\title{
WARFARE AND SOCIETY
}

Archaeological and Social Anthropological Perspectives 


\section{Index}

Abelam, Sepik 133, 182, 529

Abercromby, Lord 150

Abingdon enclosure, Thames Valley 148

Abipón 110, 401, 415, 487

Abodrites 232

Abrahám 342-343, 352, 363, 365

Abu Simbel 284

Achilles 281, 285, 424, 519521,524

Acragas, Sicily 284

action and discourse 526

action, war-related 395

Acy-Romance 287-289

Adam's Grave 144

Admiralty Islands 187-189, 191, 198-199

Adriatic coast 230

Adua 266

Adze 143

Aegean 37, 43, 283, 285, 303, $502,508,516,521,527$

Aeneas 285

Aeneid 285

Afghanistan 42, 49, 281

Africa, East 106, 113, 124, 399 , 413

Africa, southern 488

Africa, sub-Saharan 85, 213, 261

Africa, West 220, 222, 402

African political culture 261-262

Agade 43

Agamemnon 401, 518-519, 521,524

age $6-7,13-16,18,36,41,43-$ $44,51,53,55,57-65,67,69$ 73, 87, 101, 107-108, 134, $141,147,152-165,172,212-$ $213,215-216,218,221,226-$ $229,231,234-235,244,262$, $264,266,268,275-280,282-$ 283, 285-293, 295-304, 306$307,310-311,317-325,327-$ $329,331-333,335-347,349$, $353,355,357-367,369-373$, 375-379, 381-382, 386-387, $391,393-397,399,401-411$, 413-415, 417-421, 425, 429$430,446,467,473,483-513$, $515-518,521,525-528$ age grade/grading 264,268 , $399,402,404,413,521$

age, old $156,414-415,418$

age, social $71,408,415,484$

agency $59-60,70,196-197,269$,

$386,388,391,435-437,442$,

$444,475,478,484,526$

aggressive image, an asset 196

agriculture, role of 219

Aitape 203

Aksum 266

Alamanni 228, 231-232, 234, 236

Albania 453

Alcinous, Kiñg 524

Aldro 144

Alekšince 349

Alesia 286, 295, 300-301

Alexander the Great 230

Alexander, R. 31,116

Alfred the Great 232

Algeria 526

Algonquin 106, 419

Ali'i nui 248, 251-254

Aliwa 206-207

Allcock, J.B. 465

Allen, B. 173

Allia River 230

alliance $30,73,78,84,113$ $115,118,120,123-127,130$, $133,167,170,174-175,190$, $192,196,232,236,243,245$, $247,253-255,320,326-327$, $398,462,486,521,524,526$

alliance and marriage 114,125 126

alliance feasts $118,123,125$

alliance formation 113,124 $127,130,175,196,245,254-$ 255

alliances, unstable 126

allies 14, 24, 47, 50, 92, 113, $116,118-120,123-129,131$, $133,171,174,176-182,196-$ $197,244-245,247,249,389$ Alps 230, 277, 410, 489, 515, 517

Al-Qaeda 96

Altruism 25, 31-36

Amanitare, queen 284
Amazonia 111, 113-114, 118$119,128,135,386,397,404$ Ambum Valley, Enga 177, 181 Amesbury, England 154

Ammianus Marcellinus 227, 231 Amun-Re 44

analogy, principle of relational 515

analogy/analogies $31,142,212$, $276,297,299,404,415,486$, $489,505,515-516$

anarchy $78,84,86,261,270$, 453

Anatolia $42-43,51$

ancestors, proximity to 358

Andersen, S.H. 63

Anglia 157, 229

Anglo-Saxon 73, 154, 232, 279, 337, 397, 401

Angola 261

anthropologists, role of 390 , 469-472, 477

Antigua 472

Antiquity $6,41,52-53,71,102$, 159-161, 163, 165, 231, 259, 281-283, 285, 296, 298, 301, $339,420,517$

Antofts Windypit, Yorkshire 156

Antonine Wall 293-294

Apache 98, 106, 240, 320, 327, $329,403,419$

Aphrodite/Venus 523

Appadurai, A. 448

appearance, personal 15, 414, $417,484,525$

appearance, physical 414,520

Apulia 233

Aquinas, T. $38-41,44,47,53-54$

Arabic 451, 453-454, 523

Arapesh, Sepik 82, 182

Arawe Islands, New Britain 208

archaeologists, role of 390,469 472,477

archaeology, post-processual $60,64,526$

archers $147,161,355,366,372$, 418

archers' gear/archery equipment $150,348,351,361-362,418$ arctic 4,275 
Ardennes 287-288, 303

Arete, Queen 524

Argentoratum (Strasbourg) 231

Argos 519

Ariovistus 230-231, 235

aristocracy $40-41,64,154,400$ $402,503,518-519,522-524$

Aristotle 38, 461

Arklöf, Jackie 429

Armageddon 426

armament, renewal process in 495

Arminius 231, 235

armour 60, 72, 295, 298-299, $314-315,318,483-484,490$, $504,508,511,528$

arms race $107,123,133,159$

arms situation, variation 501

arms technology, changes in 267

arms, imported and adopted 495

army, acted as state police force 220

army, cohesion of 92

army, standing 40, 249

army, used to protect trade 220,225

Aron, R. 53

Arrian 38

arrowhead 58, 144-145, 147, $150,152-153,338,359,365$, $367,404,494,506$

arrowhead, armour-piercing 41 , 53

arrowhead, leaf-shaped 144, $149-150$

arrowheads, antler 361

arrowheads, barbed-and-tanged

152-153

arrowheads, bifacial 404

arrowheads, bone 150

arrowheads, bronze 361

arrowheads, flint 404, 494

arrowheads, made of lithic

material 361

arrowheads, oblong triangular

351,361

arrowheads, obsidian 404

arrowheads, pressure-flaked 65 , 404,518

arrowheads, short heart-shaped $351,361-362$

arrowheads, stone 150,358 , 361

arrowheads, transverse 404

Artayctus 296
Artemis 523

Ascott-under-Wychwood, England 144-145

Ashanti 264

Ashgrove, Fife 412

Ashton Keynes 337, 340

Asia 43, 47, 51, 142, 202, 221, 226,283

Asia Minor 283

Asia, Central 51

Assur 44-45

Assyria 43,54

Aswan 284

Athene Nikephoros 298

Athens 41, 71, 304, 527

Attalid 298

Augustine 38-41, 55

Augustus, Roman Emperor 285

Aulnat, Auvergne 300

Aunjetitz culture, late 279

Auschwitz 426

Australia 88, 132, 137, 139, $202,242,458$

Australian Museum 205

Australopithecus 305

Austria 143, 278-279, 302, 332, $346,360,371,373,417,509$ 510

Austria, Lower 279, 371, 373

authority $26,40,46,95,98$, $110,133,149,192,197,203$, 222-223, 239-241, 247, 256, $264,266,268-269,356,372$, $394,427,435,440-442,444$, $477,485,517-519,524-525$

authority, central 239

Auxilia 229, 231, 236

Avatip, Sepik 69, 132, 385-386

Aveline's Hole, England 143

awl, copper 359,418

axe, bone $357,406-407$

axe, bronze cult 493

axe, copper 407

axe, flanged 493,506

axe, polished $353,362,404$

axe, shaft hole 404-405, 407, 415,493

axe, socketed $323,493,494$

axe, stone $145-146,169,325$, $353,358,362,366,404,410$, 484,493

axe, t-shaped antler 409

axehead 415

Aztecs 223, 226

Baba Mountain 346

Babylon 43, 421
Bakassi Boys 269-270

Bakel, M. van 219

balance, establish/restore/ recreate $174-175,179,182$

Balkan metaphor 460

Balkans 13, 16, 86, 105, 228, $389,398,404,428-429,448$, 459-461, 464, 466-468, 472, 510,523

Ballomarius 231

Ballymacaldrack, County Antrim 153

Ballynagilly, County Tyrone 149

Baltic 494-495, 498

Baluan Island 187, 189-190, 193-196, 198

BaMbuti 115, 129

bands, nomadic 121

Banská Bystrica 346

Barash, D. 116

barbarian 215-216, 236, 276$277,282,290,300$

barbarism 75-76, 294

Bard, K.A. 52

Barker, F. 86

Barnack barrow, England 151

Barnes, J. 83

Barraud, C. 33

Barrow Hills 145, 152, 159

barrow, chambered long 146

barrow, long 144-146

barrow, round 144-145, 152154

Barth, F. 125, 395

Barton, Cambridgeshire 292

Baruya 118, 120, 123, 135, 184

Basa 284

Basatanya 405-406, 408-409

Basel 205

Bašić, N. 429

Basque 476

Bates, M.J. 423

Bátora, J. 341, 344, 348-349, $362,364,366,368,370,381$

battle $127,168,173,179-181$, 196, 198, 206, 219, 230-231, 233, 244, 246-247, 249-252, 254-255, 279, 282-283, 285, $289,296,298,304-309,315-$ $318,321-322,326,362,364$, $372,387,396-397,423-426$, $428,430,523$

battle of Good Friday 307, 318 battle of Towton 306,318 battle of Visby $306,316-318$ battle techniques 364 
battle, archaeological evidence 321

battle-axe $16,60,63,150-151$, $153-154,410,413,415-417$

battle-axe culture 60

battle-axes, w. blunted edge 151

battles, sea 192

Bau $65,243,246-248,254-258$

Bauman, Z. 445, 465

Bavaria 143

Bax, M. 448

Bazin, J. 217

Beaker barrow 151, 160

Beaker grave-goods 153

Beaker invaders 150, 158

Beaker network 153

Beaker period 151, 153, 506

Beaker, bell-shaped 412, 418

Beaker, drinking $410,415,417$

bear claws $357-358$

Beche-de-mer 242, 257

Becker, C.J. 63

beer 199,412

Beeston hillfort, England 158

behaviour, warlike 66,69

Beith, Scotland 155

Belas Knap 146

Belgian Congo 293

Belgrade 429, 431, 442, 445446,465

Bell Beaker (Culture) 341, 364, $387,417,419-420,493$

Bellicose rhetoric 402

Bello Gallico 227, 282-283, $289,294,298$

Bellona Island 92, 101

belt fittings $348,351,358-359$

Bena Bena 83-84, 87, 124, 136

Benedict, R. 77, 81-82, 85

Benigni, R 436

Bennike, P. 322

Beowulf $397,401,419-420$

Betsileo 212, 224-225

Bible, the 24, 45

Bicêtre Prison 292

big men 27, 79-80, 83, 87, 120, $135,138,168,171-175,179-$ $181,183,185,206$

Bigberry, Kent 292

Bijelo, Croatia $433,435,440-$ 445

Billown enclosure, Isle of Man 148

bin Laden, Osama 49,51

Birket-Smith, K. 63, 70
Bismarck Archipelago 6, 110, 201-205, 207-208

Black Sea 404

Blackfoot 106, 133, 419

Blackwood, B. 203, 205-206

Blanche Bay 202

Blok, A. $386,389,391,447$

boar's tusks $338,344,348,351$, $355,358,360$

Boas, F. 84

bodies, bound 285

Bodrogkeresztur 405, 407

body decoration 178,414

bog bodies 153, 275, 289, 295296,304

bog bodies, shaven before death 296

bog finds $153,275-276,288$ 289, 295-296, 298-299, 306, $322,492,500,508$

bogs, Danish 27ซ́, 306

Bohemia 231, 234, 337, 373, $378,387,410,413-417,421$ 422

Bohuslän $62,321,488$

Boii 230, 300

Boles' Barrow 146

bondage $285,288-290,294$ 295, 302

bondage, associated $\mathrm{w}$. ritual behaviour 289

bondage, symbolic 288, 290

bone discs $348,351,358$

bone point $143,409,494$

bones, postcranial 307, 314-317

booty $117,122,125,192,196-$ $197,217,230,232-233,295$, 298-299, 387, 486, 523

Boran 134, 265

Borneo 128, 138

Borum Eshøj 499

Boscombe Down 150-151

Bosnia $9,13,70,72,389,429$, $433-435,438,440-443,446-$ $454,460-468,510$

Bosnia Herzegovina 389,429 , 433, 447, 449-454, 461-463, 468

Bougarel, X. 451

boundary construction 268

Bourdieu, P. 386, 527

bow and arrow $243,355,361$, 370-372, 413, 494, 507, 512

Bowman, G. 448

bows $82,176,179,192,243$, $249,253,361-362,484,505$ bracers $150-152,348,351,355$, $359,361,418$

Bradley, R. 148,336

Brain, B. 305

Branč 342, 344-345, 347-349, $351-356,358-366,368,370-$ 372,382

Brandenburg 492

Brandt, E. 86

Braudel, F. 424

Braveheart 428

bravery $69,122,152,172,244$, $247,344,393,400,424,518$

Brazil $69,135,266$

Bregava River 455-457

Breiddin, Wales 158

Breisgau 231

Brennus 230

Breton 230

Brezsch Kujawski 409-410

Briar Hill 149, 159

Bridgford, S. 155-157, 509

Bridgness, West Lothian 293

Brigantes 290

Bringa, T. 450-451

Britain 6, 87, 107-108, 134, $141,143-144,146-147,149-$ $151,153-155,157-165,198$, 202-208, 263, 285-286, 288, 290-291, 293, 300, 302-304, $331,337-339,421,431,506$, 508-512

Britain, northern 146

Britain, southern 146

Britain, western 150, 208

British Papua 202

Brittany 494

Broholm, H.C. 63

Bronze Age 6-7, 13-16, 43-44, $51,53,55,57-65,69-73,107-$ $108,141,152-165,212-213$, $216,218,226,277-280,283$, $287,293,295,300,303,319$ $323,325,327-329,331-333$, $336-347,349,353,355,357-$ $365,367,369-373,375-377$, $379,381-382,391,394-395$, 401-402, 419-421, 483-513, $515-518,521,525-528$

Bronze Age, early $13,16,58$, 151, 153-154, 278, 321, 328, $338,341-344,346,353,360$, $362,364,369-370,372-373$, $376-377,381,391,394-395$, 489, 491-494, 505-508, 516

Bronze Age, late 61, 65, 72, $155,283,328,331,337-338$, 
394, 401, 491-496, 499, 502, 508-511, 516, 521

Bronze Age, middle 108, 157, $213,216,278,319-323,327-$ $328,332-333,336-339,358$, $372,381,492-495,498-499$, 506,510

Bronze Age, Nordic 63, 491, 493, 498, 501-502, 508

Bronze Age, peaceful period 151

Brough-under-Stainmore, Cumbria 291

Brown, G. 202

Brown, P. 79, 86

Bructeri 231

Brunaux, J.-L. 289

Brunn, W.A. von 506

Brøndsted, J. 60,62

Bua 243

buddhist monasteries 220

Buka 191, 198

Buleau 78

bureaucracy $93,225,256$

Burgundy 301

burial 13, 57-58, 72, 143-148, 150-154, 156, 160-163, 226, 229, 275, 279-280, 287-288, 306-309, 321-323, 326-328, $336,338-342,344,346,348-$ $349,353,355,358-364,366$, 371-373, 376, 378-381, 387, 394, 404-405, 408-417, 420, $422,485,490-491,494,499$. $502,512,516,519-520,525$ 526,528

burial and personal equipment 519

burial custom $341,362,371$, $405,409-410,417$

burial deposit 146

burial finds tell of social elite 13, 346, 371

burial position 308,414

burial practice $58,346,353$, 360

burial ritual $372,502,516,519$ 520

burial site $307,322-323,340$, 353, 360-361

burial, abnormal $58,369,373$, $376,381,487$

burial, Beaker 151

burial, cremated 153

burial, crouched position 287 , 405

burial, females 418 burial, inhumation 152,156 , $306,322,346,489,492-494$, $499,502,511,516$

burial, left-hocker 409-410, 412-414

burial, males $144-145,234$, $405,408,410,415,418$

burial, multiple 143-144, 369, $373,378,380$

burial, right-hocker 409-410, 413-414

burial, wetland 145

burials, used to mark differences 502

burials, violent and ritual aspects of 378,380

Burl, A. 156

Burma 220, 226

Butoni 231, 246

buttons, v-perforated 418

Byzantine 233

C-14 307-308, 317

Cachtice 362

Caesar, Julius, Roman Emperor $38,215,227-228,230-231$, 235, 282-282, 286, 289, 292, 294-295, 298

Cairn 145, 151-152, 161, 164, $279,323,328$

Caka mounds 502

Cakaudrove 243

Cakobau 247, 254-256, 258

Cakonauto 246, 255

Calabria 233

calcification of bone 324

Caldey Island 143

Callis Wold 144, 154

Calypso 523

Cambridge, England 153

Cameroon 222, 226

campaign 116, 230, 244, 253, 290

cannibalism 75, 82, 192, 291

Cape Gloucester 202-203

capitalism 47

captivitiy and transformation of being 283

Caratacus, British freedom fighter 290

Carn Brea, Cornwall 163

Carneiro, R.L. 52, 89, 97-99, $212,219-220,238-242,248$, 257

Carolingian Empire 232

Carolingians 223

Carpathian Basin 410
Carpathians 346, 363

Carpentras, Vaucluse 293, 298

Carrier, A. 191

Carrier, J. 191

Carshalton 157, 159

Carthage 230, 235

Cartimandua 290

case study $15,185,199,226$, $270,322,329,353,387,413$, 466,489

Cassandra 523

casualties $48-49,83,114,153$, $213,218,243$

categories of evidence 505

cattle $71,125,147,158,228$, $267-268,307,328,342,344$, $372,399,411$

Cauca Valley 101, 257, 401

causal elements/factors 212$213,219,265$

causality 217-218, 222, 225

cause and effect 212, 218, 221, 389,474

causes, demographic 53

causes, economic 222

causes, ideological 222

causes, politico-strategic 222

Cauvin, J. 41

Celtiberian 300-301

Celtic/Celts 227-228, 230, 234$235,276,300-301,323,500$

cenotaphs $13,358-360,368$, 370-372, 404

centralisation $98,150,214$, $220,238-240,254,256,399$, 449

centuria 229

Černý Brod 349, 362-364

Cerný, V. 421

Červen y Kameñ 346

Chabal, P. 264

Chagnon, N. 32, 35, 116, 118, 132

chain-gang 283, 292-293

Chalcolithic 410

Challenger Expedition 191

change, dynamics of 371

change, political within context of war 241

Chanson de Roland 424

Chansons de Geste 387, 424

Chapman, J. 63, 404, 507

Chatti 231

Cheddar Man 143, 164

Cherusci 231

Chewong, Malaysia 32 
Cheyenne 115-116, 137, 320, $327,329,399$

chief, embodiment of society 252

chief, Hawaiian, religiously sanctioned 253

chief, paramount 244, 251-254, 256,401

chief, ritual $256,326,501$

chief, warrior 326,501

chiefdom $63,89,99,101,225$ 226, 238, 241-242, 246-249, $255,257,326,527$

chiefdom, complex 248, 257

chiefdoms, Polynesian 95, 523

chiefs $6,64,71,81,92,100-$ $101,137,142,158,162,192$, 218-222, 224, 226, 232, 237, 239, 241-249, 251-259, 264, 294-295, 320-321, 326, 397, 401,500

chiefs, Fijian 92, 255

chiefs, Hawaiian 248, 254-255

chiefs, power of 245,257

chieftain $64,289,292,295$, $297,348,356,358$

Chilbolton, Wessex 151

Childe, V.G. 27, 59-63, 66, 491

childhood $63,146,312,377$, 414-415, 417-418

Chimaltenango 471

Chimbu 182

China 51, 234, 236, 242, 248, 303,461

chivalry, Christian 424

Chlopice-Veselé 343-344, 362, 382

Chnodomar, king 231

Christian II, King of Denmark 307

Christianity $39,45,184-185$, $233,255,328$

christianity, converting to 233 , 255

chronology $160,343,353,361$, 363

Chute 144, 163

Cicero 38,41

Cimbri 230, 294

Cimbrian 297

Circe 523

circumscription 52-54, 98-99, $219-223,225,240$

cire perdu technique 494

citizen-armies 425

city state $42-43,92,214,105$, $214,297,398,516-518,528$ city states, Greek 214,297 , 516-518

civil society $31,39-40,47,428$

civil War, English 155

Civilis, freedom-fighter 296

civilisation $10-11,26,28-29$, $32,34,50,64,70,81,106$, 233, 424-425, 441-442, 461, $464,467,470$

civilisation, American 81

clades variana 231,279

Claessen, H. 89

clans, agnatic 83

Clark, G. 63

class $60,101,147,150,220$, $231,237-239,248-249,312$, $321,348,368,371,380,396-$ $397,401,427,451,471,517-$ 519,527

Clastres, P. 106, 109, 133, 396, $403,419,448$

Claudius, Roman Emperor 290

Clausewitz, C. 28, 55, 86, 391, 478

Cnip, Scotland 153

coexistence $390,448-449,451$ $452,454-457,459,464-465$, 467

Cohen, R. 217, 219-220, 223

Cohodas, M. 478

Cojtí Cuxil, D. 478

Cojtí Ren, A. 478

Colby, B. 473-474, 476

cold War 24-25, 47-49, 107, 261,468

Coldrum, England 146

collective representations 25

collective tombs 278

collectivism 398

colonial change 204-205, 208

colonial histories 204

colonial histories, studying 204

colonial oppression 262,472

colonial pacification 6,187

colonial power $14,17,204,208$

colonialism $6,66,139,191$, 201, 203-205, 207-208, 257, 262-263, 266, 470, 472, 475, 479

colonialism, understanding war 201

colonialists 390,469

colonisation $110,188,213$, $228,232,402,444$

Člović, I. 431

Colson, E. 121

Colt Hoare, R. 146, 156 combat $6-7,80,90,114,142$, $151,153-156,167,176,192$, $234,253,305-307,309,311$, $313-315,317,319,321,331$, $336,338,360-362,364,368$, $372,423,425-426,428-430$, $486,488,498,506-507,511$

combat victim 338

combat, close/face-to-face 114 , $253,314,319,322,360-362$, 364,372

Commodus 231

communism $47,82,451,459$

companionship 68, 399-401, $415,419,488,521,525$

comparative procedure 417

compensation $93,115,123$, $127,132,174,177,179,264$, 281,285

compensation payment 132 competition $17,26,43,60,67$, 77, 116-118, 132, 141-143, $153,170,181,184,195-198$, 222, 241-242, 262, 267-268, $371,400,428,519$

composite bow $357,412,418$

concepts of culture and history, defined by outside powers 390,476

conceptual framework 13,28 conflict $6,12,24,29,31,35$, $39-40,42-44,46-54,60,63$, $67,71,73,77,83,90,93$, $101,113-115,118-121,127-$ $130,134-139,141-144,148-$ $156,158,163,168,173-174$ 176-178, 182, 184, 187, 195, $197,212-213,217-219,222-$ $223,225,228-229,238,241$, 257, 261-271, 301-302, 320, $322,326-327,329,360,370$, $386,421,426,430-431,434-$ $435,438,441,443,445-446$, $464,466-467,472,476$

conflict management 93,119 , 136

conflict resolution 115,121 , 262-263, 266-267

conflict, characterising social interaction 115 conflict, civilisational 441 conflict, factors behind 212 conflict, global 47-48 conflict, ideological $24,47-48$ conflict, internal $114,173,182$, 370

conflict, national 441 
conflict, pre-state 218

conflict, result of population growth 219

conflict, scales of 213

conflict, social reproduction of 266

conformism $7,388-389,433$, $435,437,439,441-443,445$

Congo 261-262, 269, 293

Connell, R.W. 429-430

conquest $37,39-41,43-46,50$ $53,60,89-90,97-101,110$, $128,136,184,198,214-215$, 220-221, 233, 237-242, 244, 247-249, 251-256, 262, 264, 270, 284-285, 294-295, 297, $302,317,402,469,513$

conquest and social change 247

conquest theories $98-99,240$ 241

conquest, result of war 214

consumption, alcohol 397, $410-411,418$

context approach 99, 240-241, 255

contexts $7,15,51,67,70-71$, $90,99,107,113,119,125$, $128,138,168,182,238,241$, $264,266,275,283,285,291-$ $292,302,395,402-403,436$, 452 , 489, 491, 493-494, 498$501,507-510,517-518$

Cook, James 242, 248

Copán Ruins 471

Copenhagen 12, 70-71, 101, $159,226,236,280,306,309$, $318,328-329,339,420,466-$ $467,490,502-504,513,527-$ 528

copper $7,18,150-151,153$, $341,344-346,348,352,357-$ $360,362-364,370-372,386$, 393-395, 397, 399, 401, 403$415,417-419,421,484,493$, 505, 507

Copper Age $7,386,393,395$, $397,399,401,403-411,413$, $415,417,419,421,507$

copper industry $341,344,364$ copper ore, access to 370,372 copper production, control of 371

Corded Ware (Culture) 15, 343, 387, 393-395, 404-405, 410, $412-413,415,417-419,422$

Cordy, R.H. 257
Cornwall 148, 163, 258

Corsica 448

cosmology $36,391,467,473-$ 475

cosmology as sense-making structure 473

Costa Rica 34

Cotswolds 160, 337

Cotzal 473-474

counterdiscourse $13,17,449$, 465

Country Antrim, Ireland 155

courage $38,75,117,122,344$, $387,423-425,430$

Court Hill, England 153

cowardice 122, 424

Crawford, O.G.S. 146

cremation 152-153, 156, 285, $394,494,498-499,502,516$

cremation graves 494,502

Crete $43,285,304,523$

Crickley Hill, Gloucestershire 147

crisis $102,267,270,296,387$, $402-403,427$

crisis and external pressure 15 , $387,402-403$

Crno, Croatia 434, 445

Croatia 388, 429, 433-435, 439$440,442,444-446,467$

Crow Creek massacre 300, 304

crusade 426

Cuba 281, 304

cults $41,109,168-169,171$ 173,185

cultural change $63,82,187$, $213,265,269,393$

cultural continuity $63,390-391$, 473,478

cultural differences 64,390

cultural evolution 141-142, 184

cultural knowledge as objectified systems of thought 477

cultural knowledge as practice 477

cultural norms and ideals 117

cultural relativism $27,264,271$

culture $6-7,11,14-15,18,25$ $26,28,31-36,55,57,60-61$, 63-64, 69, 71-73, 76, 80-86, 92-93, 102, 106, 117, 122, $134-139,142,158,161,164$, 185, 190-191, 204-206, 208, $213,225,230,232,236,259$, 261-266, 268-270, 277-279, 282-283, 285, 290-291, 295, $297-298,301,305,338,340-$
$344,346-349,353-356,358-$ $368,370-373,378,381-382$, $385-387,390,393-395,399$, 404-405, 409-410, 412-413, $415,417-422,427-431,445$, $448,460-461,467,469-471$, $473,475-479,481,483-488$, $490,492-494,496,498-504$, $506,508,510,512,515-516$, 518-520, 522-526, 528

culture and agency, theory of 269

culture as ideology 471

culture as lived experience 471 culture vs. race 81

culture, decisive factor 213

culture, feminine 82

culture, macro-regional 417

culture, masculine 82

culture, politicised version of $390,452,469,477$

culture, ressource for conflict resolution 263

culture, role in conflict 265-266

culture, source for violent expression 263

culture, what is 263

cultures of violence 213,262 , 265-266, 268, 270

cultures of violence, outcome of war 213, 265-266, 268

Cumbria 291

Cunnington, M.E. 146

Cunnington, W. 146

cyborg, postmodern warrior 430

Dacian 294-295, 297, 300

dagger $42,145,150-151,153-$ $156,192,195,249,294,319$, $323,348,352,355,358-364$, $366,370-371,376,394,404-$ 407, 409-410, 418, 483-485, 493-494, 499, 505-508 dagger, Abrahám type 352,363 dagger, boar's tusk 404-405 dagger, Branč type 352,363 dagger, copper 359,404 dagger, flat-tanged copper 418 dagger, flint $145,150,394$, $404-407,409,493,505-506$ dagger, miniature 363 dagger, Nitra type 363 dagger, rounded grip tongue 352,363 dagger, Šal'a-Veča type 352 dagger, triangular 363-364 
dagger, Výčapy-Opatovce type 352,363

dagger, willow-leaf 352,359 , 362-364

daggers, Arreton series 506

daggers, associated w. males 150,153

daggers, inappropriate as weapons 153

daggers, use in hunting 394, 507

Dahomey 223, 225

Daloz, J.-P. 264

Damm, C. 60

Danebury, Hampshire 282, 286

Danegeld 232

Dani 114-115, 118, 133, 136

Danube River 231, 342, 404, 417,510

Dark Age 495

Dartmoor 157, 161, 336, 339

Darvill, T.C. 145

Darwin, C. 31

darwinist $32,133,425$

Das, V. 465

Dawson, D. 142

De Coppet, D. 33

de Waal, F. 32-33

death house $346,348-349,355$ 356

death, attitude towards 516

death, cause of $144,152-154$, $156,313,325,376,405-406$, 488

decapitation $153,285,295$, 298-299, 308, 322

Decebalus, king 300

decentrality 105

defeat $39,41,45-48,50-51,54$, $92,99,113,120,123-125$, $131,230,243,247,262,282-$ $284,290,294-295,298,301$

defenders 150, 244-245, 278-279

defensive equipment 339

defensive means, absence of 502

dehumanisation 281-282

Demarest, A. 470-471

Denmark 4, 6, 11, 63, 65, 7173, 226, 297, 300, 305-307, 310-312, 317-318, 321, 338, $420,464,490,492,496,498$, 501, 503-504, 508-509, 513

dental attrition $310-311,325$

Dentan, R. 76, 130

dentition 312

dependency and fellowship 401 depopulation, different reasons 229

deposition 7, 151, 266, 287$288,298-299,304,332$, 336$337,339,485,493,498,500$, 505-511, 513

deposition in wet places 511

deposition of bodies 288, 337

deposition rules 498

deposition, context of 505-506, 509

deposits, ritual 148, 485-486, 489

deposits, secondary 328

Derventa 441

desecration 328,360

desecration, of war heroes 328

Deskford 299

Didmarton 337

difference, marker of 391

differences, familiarisătion of $452,459,464$

differences, narcissism of minor 386,447

differential reception 412

dimorphism, sexual 414

Dinka 126, 263, 265

Dinorben, Wales 158, 161

Diomedes 519-520

Dionysius, tyrant of Syracuse 230

Dionysus 296

Dirks 155, 160, 510

discourse $7,10-11,15,18,57$, $62,77-79,85-86,134,214$, 261-263, 265-267, 287, 328, $383,385-391,393-398,400$, $402-404,406,408,410,412$, $414,416,418,420,422,424$, $426,428,430,434-446,448$, $450,452,454,456,458,460$ $462,464,466,468,470,472$, $474,476,478,484,515-516$, 526

discourse and action 526

discourse and agency 388

discourse and everyday life 389,439

discourse as signification 396 , 403

discourse, dominant 390

discourses, authoritative 389 , 437-441, 443-444

disease $136,163,203,207-208$, $305,312,318,328-329,339$, $402,405,469,503$ disorder $29,68,79,83,94,264$, 270

displacement $183,346,433$, $437,442-443,473,476$

dispossessed, voice of 477

diversity $134-135,165,212$, $262,264,267,299,457$

Dizi 266-268, 270

DNA analysis 279

Dobu 78, 81-82, 86

Domesday Book 337

domestic production, control of 371

domination and coercion 402

Donegore Hill, County Antrim 148

Dorchester 151, 164-165, 338, 340,492

Dorchester-on-Thames, Oxfordshire 156

Dorset 147, 159, 161, 163-165, $337,339-340$

doves 27, 66-67, 115, 117, 127

DR Congo 262

Drekete 246

dress accessories 414, 417-418, 485

dress finery 409-410, 413-414

Drewett, P. 149

drilling 92

Drumman More Lake, County Armagh 156

Dubrave 462

Due, O.S. 516,527

Duggleby Howe, Yorkshire 145, 150

Duke of Yorks 203

Dumas, Alexandre 425

Dumont, L. 25, 30, 33

Durham, W. 132

Durkheim, E. $31,35,465$

durkheimians $30-31$

Earle, T. 220, 223

earthen mound 410

East Anglia 157

East Germany 498

ecological crises 63,213

economic structures, different 228

economy, domination of the 224

economy, shift in $370-371$

egalitarian $6,16-17,25-26,59$, $63,98,108,111,122,133$, $167-171,173,175,177,179-$ 
$183,185,221,240,243,321$, $402,518-519,526-527$

Egypt 41-43, 51-52, 54-55, 284, 295-296, 301-303

Egypt, New Kingdom 284, 295

Egypt, Pharaonic 302

Egyptians 41, 43-44, 424

Eibl-Eibesfeld, I. 31

Ekagi-me 120

Elbe River 229-232, 234, 509

Eleutherna, Crete 285

elite $13,15,18,61,64,237$, $242,254,256,262,269,306$, $326-327,346,355-358,360$, $368,370-371,394,399-400$, $411,414-415,417-419,428$, $473,485,507,511,517-519$, $521,524-525$

elite's point of view 524

elitism 396

elitist culture, universal components of 525

Elysium 423

Ember, C. 76,120

Ember, M. 120

emigration 228-229, 231

empire $38,42-44,95,105,135$, $192,212,215,223,228-229$, 231-235, 264, 295, 402, 470

enclosure, causewayed 143-151, 507

enclosure, ceremonial 149

enclosure, fortified 148

enclosure, hilltop 148

enclosure, palisade(d) 149, 151, 409

enclosure, ringwork 157

enclosure, ritual 149

enclosure, unfortified 148

enclosures, main role of 157

endogamy 415

enemy heads, taking of/

head-taking 298, 300

eneolithic $341,344,348,361$, 364,507

Enga Province 184

Engels, F. 31, 402

England 47, 135, 143-146, 148161, 163-164, 184-185, 229, $232,234,279-280,282,286-$ $289,291,299,331,339,362$, 423

England, central 145, 151, 157

England, central-southern 289, 299

England, eastern 144, 148-149, $151,153-154$
England, northern 144-145, 151-153, 157-158

England, northwest 153

England, southeast 146, 148, $151,155-158$

England, southwest 143, 155

England, western 144, 146, $150,153,158$

enlightenment $29,31,263,424$

Entremont, southern France 297,300

environment $17,26,28,32,55$, $83,85,91,94,107,116-118$, $120-122,125,129-130,133$, 135-137, 141-142, 161, 164, $180,185,187,239,279,335$, $382,439,451,512$

environment, anarchic 120

environment, natural 32

environment, warlike 28,83 , $116-118,120,122,125,129$, 133,187

equality $33,62,129,169-172$, 179-180, 182-185, 265, 397, $401,415,424,430,452,477$, 518-519, 524

equality, ideology of 415,519 , 524

equals, first among 401, 518519

eques 231

Erskine, J.E. 257

Ertebølle 63, 143

essentialisation 469,477

Essex 148, 159-162, 303

Ethiopia 213, 264, 266-268, 270-271

ethnic cleansing $282,302,389$, $443,446,456,465,467$

ethnic identification 389

ethnic nationalism $\quad 388-389$

ethnic resurgence 475,479

ethnicity $18,139,236,270$, $395,415,420,445,449,451$, 457-458, 464, 466-467, 479, 485

ethnogenesis 227-229, 231-232, 234,236

ethnohistorical perspective 6 , 167

ethnohistory $72,111,135,162$, $329,403,479$

Etton 149,163

Eudusii 230

Euphrantides 296

Eurasian steppe 62
Europe $6-7,13,16,18,24,27$, $38,44-47,59,62-65,71-73$, 77-78, 82, 93-94, 105, 108, $139,142-143,145,150,153$, $155,160-164,211-212,214-$ $216,227,230,234-237,242$, 257, 259, 262-263, 268-269, 276, 278-280, 285-286, 289, $293,295,298,300-301,303-$ $304,306,329,331,337-340$, 344, 346, 361, 363, 369, 372, $381-382,386-387,389,391$, 393-395, 397-399, 401-405, 407-413, 415, 417-421, 427 . $428,448,453,459-461,464$ $465,467-468,485-487,489-$ 490, 492-493, 495, 502-504, 507-513, 515-516, 525

Europe, central 15-16, 59, 105, $150,227,230,233,278,337$, $344,346,361,363,372,381$, $387,393,404,408-410,412$, $417-418,485,487,492,494-$ $495,508,510,516$

Europe, northern 105, 143, $296,298,339,387,401-402$, $410-411,460,487,489$

Europe, protohistoric 215-216

Evens, T. 126

everyday life $17,68,72,243$, $320,389-390,411,431,437$, $439,443-444,446-448,451$ $452,457,459,467,478$

everyday life, reconquering 443-444

evolutionary advantage 26 evolutionary biology 35 evolutionary economics 133 evolutionary psychology 31 , $35-36,141,158,160$ evolutionary theory 27,135 , $137,141,160$

evolutionism 84, 101, 226, 257 excavation 147-149, 151, 154, 159-164, 258, 303, 307, 313, $323,328-329,331,334-336$, $339,373,502$

exchange $6,14,17,25-27,29-31$, 33-36, 64, 68, 73, 77-81, 83-84, $86-87,93,95,98-99,108,110$, 114-115, 118-120, 123-125, $130,132,137,139,149-150$, 168-174, 176, 178-182, 184$185,187-193,195-199,201-$ 202, 204-208, 215, 221-222, 241, 255-256, 263, 329, 386, $410,502,520,526,528$ 
exchange networks 95, 149, $168,171,178,180,189-190$, 206-207, 256

exchange, alternative to war 120 exchange, ceremonial 79,119 , $168-169,178,190$

exchange, importance of 191

exchange, key element in Manus culture 190

exchange, marriage 25,114 , 119-120, 124, 172

exchange, motivation to engage in 207

exchange, role of individual in 190

exchange, symbolic 30,34

exchange, traditional systems of 110

execution, ritualistic 152,286

expansion 18, 24, 38, 42-44, 46-48, 50-51, 60, 91, 105-

$106,109-110,119,126,128$, $139,169,207,220,230,235$, $249,263-264,341-342,371$, $402,420,520,527$

expansion, territorial 24,38 , $44,46-48,50-51$

Eyre, C. 52

Fabbro, D. 133

Fagan, B. 131

fait social total $25,30,34$

family $18,30,36,40,66,76$, $92,168,171,183,189-190$, $196,202,207,221,224,228$, $247,257,264,326-328,331$, $339,347,350,355-356,358$, $361,371,413,427,437,440-$ $442,451,454-455,458,460-$ $462,466,477,479,486,492$, $521-524$

farming $42,57,148,226,228$, $235,266,326,335,344,419$

fear $38,78,85,117,122,124$, $126,130-131,141,171,174$, $182,196,242,266,268,276$, 281-282, 290, 301, 321, 434, $441,462,473$

Feil, D.K. 168

female clubs 398

female domain 522

female, high-ranking 247, 252, 256-257, 411, 417

female, high-status, peaceful 414

female, low-status 414 female/women, young 145, $153,245,296,379,414$

femaleness 62,415

femaleness, peaceful 62

feminine, militarisation of 430

femininity 82

Fengate 144, 163

Ferguson, R.B. $67,109,114$, $119,132-133,142$

Fesques, Seine-Maritime 289

feud 113-115, 132, 135, 277

Field Museum, Chicago 205

fight leaders 181

fighting (and feasting), element in maintaining lapan status 189

fighting, demonstration of potency 488

fighting, intra-village 92

fighting, ritualised 61

Fiji $6,13,16,92 ; 95,101,214-$ $215,237-238,242-245,247-$

$249,253-259,278,401$

Finley, M. 516, 520, 527

firearms, introduction of 110 , 133

firearms, sale of 208

firearms/fire weapons 17, 93, $110,133,192,197,202,207-$ $208,214,243,248,255-256$, $268,427,452-453,493,507$

fishermen, sedentary 116,121 , 129,132

Five Knolls, England 145

followers, mobilisation of 402

Fontijn, D. 218

Fordington Farm barrow, Wessex 152, 159

forensic analysis 277, 379

Fornander, A. 253

Forsayths family 202

fortification $52,57,96,167$, $224,229,233,243-245,247$, $251,253,255,295,395,404$, 487

fortifications and territorial marking 487

fortifications, nodal points 487

fortified settlement 148

forts, hilltop 224

Fortune, R. 81-82

Foucault, M. 283, 292-293, 296, 302

fousive 98,240

Fox, R. 31

France 47, 92, 137, 231-232, $234,243,247,257-258,263$,
293, 297-298, 300-301, 425,

506

Frankenstein, S. 71,527

fraternal interest group 397

fraternities 397

freedom 39-40, 231, 265, 291, $294,475,519,524$

French Revolution 45, 388, 425

Freud, S. 386, 447

Fried, M. 96, 98, 221, 238, 240

Friesia 232

Fuller, J.F.C. 50

function $7,14,63,73,82,95$, $106,118,126,157,159,167$, $184,187,191,219,226,229$, $234,242,246,326,340,361$ $362,387,404,439,444,448$, $484,486,490,497,505,507$ $508,511,527-528$

function, independent of material form 484

Funen 492

funerals $30,328,394,419,485$, 519

funerary custom 413

funerary domain $18,394,402$, 408,485

funerary etiquette 18

funerary monument 394,485

funerary practice, orientation of the body 346

funerary ritual 146,355

Funnel Beaker people 63

Funnel-necked Beaker 404

Fussell's Lodge 145-146, 150, 159

Fyfield 144

Gaimar IV 233

Galatians 298

Galgedil 494, 503

Gallay, G. 506

Gallic Wars 294

game theory $121-122,131,133-$ 134

gang-chain 292-293, 301

gangs, modelled as warrior societies 427

Gardner, W. 158

Garton Slack 338

Gat, A. 132

Gaul (Gallic) 110, 215, 230, $232,235,276,282-283,285-$ 287, 289-291, 293-295, 297301

Gaul, Cisalpine 300

Gazelle Peninsula 202-203 
Gefolgschaft 15, 64, 214, 216, $223,228,229,397,400,403$, 521

Gelduba/Krefeld 279

gender $7,14-15,18,62,69,72$, $82,87,111,124,162-163$, $199,207,225,268,296,298$, $303,310,321,346,387,395-$ $397,399,404-406,409-411$, 413-415, 417-421, 423-424, 430-431, 451, 467-468, 484$486,489,515,517,519,521$ $525,527-528$

gender and rank 387, 413, 415, 417,419

gender differentiation 18,404 $405,409-410,414,418,420$

gender distinction 418

gender equality 424,430

gender stereotype 410,419 , 522

gender, biological sex 414 gender, social 484

gendered life course 405, 421

gendered objects 410

gender-specific position 410

genocide $64,67,282,302,402$, $445,466,479$

Gent, G. 334

Georgieva, C. 452

Gerloff, S. 506

German New Guinea 202, 208

German Wars of Liberation 425

Germany $46-47,71,75,88$, $143,162,202,208,230,232$, 234-235, 263, 270, 289, 299, $303,306,338,346,362,368$, 421-422, 435, 461, 496, 498, 506-508, 510, 527

Germany, eastern 506-507

Germany, north 289, 508-509

Germany, southern 496

Geronimo 98, 240, 403, 419

Gerumsberg 493

Gerzon, M. 429

Gettysburg 426, 428

Gibson, A. 152

Gibson, J.W. 428

Giddens, A. 90, 94, 96, 397, $478,484,526$

Giddings, R. 173

gift $5,25,29-31,33-34,36,64$, $68,77-81,83-85,87,114$, 118-120, 124-125, 132, 190, $196,199,291,448,466$ gift exchange $30,33,64,68$, 79-81, 83-84, 114, 118-120, $124-125,132$

gift, means of social control 81 gift, pacifying 80

gifts to the gods $394,408,486$

Gilgamesh 7, 387, 423-425, $427,429,431$

Gimbutas, M. 62-63, 66

gladiator 428

Glenquickan, Scotland 145

Gleser, G.C. 311

Glob, P.V. 60,62

Gloucestershire 12, 147, 159, $164,331,337,340$

goddesses $38,519,523$

Godeffroy und Sohn 202

Godelier, M. 120

gods $24,37,41,44,46,54-55$, $71,78,160,244,246,251-$ $254,258,285,289,296,298-$ $299,303,394,408,466,474$, $479,486,488,519,523$

Gog Magog Hills, England 153

Gombe Reserve 33

Gommern, Saxony-Anhalt 229

Goodale, J. 207

Gordon, R. 119

Goths 215

Gotland 306, 310-311, 317, 494

Gournay-sur-Aronde (Gournay) $234,276,299-300$

grave robbing/robberies 360 , 370

Gradić, Bosnia 441

Graeco-Roman 286, 298, 300

gramscian 436

Grand Chaco 16, 110, 401-402, 415,487

Grandtully, Scotland 152

Granhammar, Sweden 492

grave finds, rank interpretation of 501

grave, female 310

grave-goods $153,159,229,287$, 289

grave-goods, absence of 287

graves w. weapons, interpretation of 216, 502

graves, best documented contexts 500

graves, chiefs' 500

graves, full-length $\mathrm{w}$. swords 498

graves, megalith 306

graves, plundered 358 graves, spatial arrangement of 348

graves, warriors' 358

Gray, C.H. 429

great men 120, 135

Great Plains tribes 399

great-ape societies 26

Greece 24, 44-46, 51, 214, 235, 401, 490, 505, 511, 516-518, 523,527

Greece/Greek, ancient 24, 4546, 281-282, 284-285, 289, 296-297, 401, 505, 516-518, 527

Green, H.S. 152

Gregor, T. 129-130

Gregory of Tours 227

Greuel, P. 126

Greyhound Yard, Dorchester 151,165

Grinsell, L.V. 156

group for itself 396,404

group in itself 396

group size $123,125,127,133$

grove deity 290

Guantanamo Bay 281

Guatemala 390, 468-469, 471$473,475-476,478-479$

Guatemala City 475

Guatemalan War 472

guerrillas 427, 473-475, 479

Guichard, V. 292

guilt $427,443,473-474$

Guinea 5-6, 12, 14, 16-17, 27, $68-69,73,75-88,93,101$, $107-108,110,113-114,118-$ $120,124-125,128,135-139$, 167-169, 182-185, 187, 191, 198-199, 201-204, 206, 208, $261,385-386,397,404,466$

Guiscard, Robert 233

Guiscard, Roger 233

Gunawardana, L. 220

Gundomad 231

guns see firearms

Guttmann, E. 157

habitus $263,266,269,415,457$

Habsburg empire 429

Haedui 230

Hagen 79,87

Hagenbach 232

Hagesteijn, R. 221

Hahl, Governor 202-203

Haile Sellassie, Emperor 266

hair, liminal substance, treatment of 298 
Hajduk tradition 429

Hajer, M. 388, 439

Halberd 153, 363, 506-508

Halberds, occurence in hoards 506-508

Hallpike, C. 121, 130, 223

Hallstatt Culture 502

Hambledon Hill, Wessex 144

Hamburg 136, 198, 232, 236, 271,503

Hamilton, S. 158

hammer axe $353,357,408$

hammer axe, small heartshaped 362

hammer axe, w. facetted butt 362

hammer picks 362

hammer, grooved 362

hammers 361-362, 493

Hammurabi 43

Hamn, Sweden 287, 300

happy hunting grounds 423

Harald Klak 232

Harbison, P. 507

Harborough Rocks, Derbyshire 144

Harding, A. 154-156, 326-327

Harris, M. 118, 217

Harrison, L. 265

Harrison, S. $68-70,77,80-81$, $85,91-93,122,132-133,183$, $385-386,391,448$

Hartlepool Bay 145, 164

Harudes 230

Hasanlu 279-280

Hassleben-Leuna 232, 236

Hastrup, K. 326

Hawaii $6,13,16,95,214-215$, 237-238, 242, 248-249, 251259,401

Hawks 27, 66-67, 115, 117, 127

Haywood Cave, Somerset 145

Hazleton, England 144-145

head-hunting $75,79,82,114$

headman 114, 203, 320, 407

heads, severed 297-298, 300301

healed fractures $145,151,360$, $366,377,414$

Hebrew 24, 40, 44-45

Hebrew Bible 24, 40, 45

Hector 527

Hedeager, L. 60-61, 66, 400

Hegel, G.W.F. 24, 39-41, 45-46, $50,53-54$

Hegemonic 436

Heidenreich, C.F. 218
Helbling, J. 69

Helena 523

Helman Tor, Cornwall 163

helmet $316,486,517,520,527$

helmet's cultural identity 520

Hembury Enclosure 147

Henry VIII, King of England 318

Hequetai 401, 521-522

Hera 523

Herder, J.G. 263

Hernádkak, Hungary 338

hero worship, individualising 516

hero/heroine $60,66,70,173$, $175,179,285,287,321,327-$ $328,423,425,427-429,488$, $515-524,527$

Heuneburg 494

hidden transcripts 391,436 , 442,446

Hierakonopolis 284

hierarchies, weak 108, 182

hierarchisation 214,220

hierarchy $16,94,97-98,106$, $108-109,114,167,183-184$, $198,214,217,222,224,240-$ 241, 246, 251-252, 254-256, $264,284,395,400,402-403$, $427,477,498,515-516,518-$ $522,524,526$

hierarchy, predetermined 518

Hill, E. 283, 295

Hill, R. 472

Himmerland 494

Hindwell, Wales 151

hird 521

Hiroshima 426

historical development, individual factors in 214

history of war studies 9

history, end of 40

Hittites 43

Hjortspring boat 338

hoard deposit 498, 506, 509510

hoard, sacrificial 408

hoards $70,155,319,326-327$, $394,408,486,489$, 495, 498$500,506-512$

hoards, interpretation of 500

Hobbes, T. 121, 218, 263, 337, 419

Hochschild, A. 293

Hodder, I. 68

Hoffmann, S. 46

Hog Cliff Hill, Wessex 157
Hohmichele 494

Holešov 342-344, 351, 353, 361-364, 370-372

Holland 492

holocaust $465-466$

Homer 7, 14, 38, 41, 44-45, 48, $51,214,281,285,296,303$, $397,400-401,403,424,431$, $483,488-489,502,505,515$. $517,519,521-528$

Homer as analogous social context 516

Homer, gender in 522

Homer, material culture and society 519

Homeric poem/epic 43, 283, $397,401,403,424,488-489$, $515-517,521,523-526$

Homeric society $515-516,518$, $520,523-525,527-528$

Homeric society, ideology and social organisation in 518

Homeric society, war and aristocratic warriors 520

Homer's epics, social and material world of 489

homicide $23,90-91,115,176$ $178,328,487$

Homo duplex 31, 33-35

Homo oeconomicus 31

Honduras 471

honour $15,24,38,45,49,64$, $158,161,173,235,243-244$, $253,281,285,355,385,387$, $421,424,429-430,504,519-$ 521,523

hoplite 398

Horik I 232

horses $15,110,158,228,233$, 299-300, 402, 410, 487

Horton Camp 337

hostage $288,290,474$

household 170-172, 174, 180, $229,247,320,451,477,522$, 524

Howard, M. 77

Huldremose, Denmark 296

Huli $86,133,182,184$

human body $34,282,287,295$, 395,484

human history $25,42,50,89$, $134,237,423-424$

human nature $23-25,29,31$ $32,34,36,39-40,50,52,54$, $141,165,184,465$

human nature, duality of 25

humanitarian aid 434 
Hume, D. 45

humiliation $172,174,181,247$, $250,265,270,276,281-283$, $285,288,290,292-295,298-$ $299,302,462$

humiliation, iconographies of 293-294

humiliation, ritualised, public 292,302

Hung bodies 300

Hungarian Plain 370, 405

Hungary 338, 406-408, 508

Huns 215

hunters and gatherers 26,76 , $107,115,119,121,129-130$, $132-133,142,218,266,424$

hunting $30,41-42,57,63,68$, $118-119,130,134,137,156$, 168-169, 176, 185, 205-206, $320-321,326,344,361,394$, $415,423,484-485,493,506$ 508

hunting and gathering 42, 63, $130,137,168-169$

hunting, prestige activity 485 , 507

Huntington, S. 265

Huron 218-219, 226

Hus, Manus 190

Hussein, Saddam 48

Hutu 228, 391, 447, 467

Hyksos 43

Hynes, S. 423, 426

Iban 114-115, 119, 124, 128, 136,139

Iceland 326

iconographic presentations, art and rituals 488

iconography $57,284-285,287$, $289,292-296,298,301,488$

iconography, war- 57, 284, 292 ideals of aggressiveness 398 identification, localistic 451 , 454

identities, ascriptions of social position 385

identities, creation of 386

identities, war-specific 385

identity $7,11,13-15,17-18,30$ $34,50-51,59,64-66,69,81$, $85,139,142,164,196,211-$ $212,215,236,247,263,266$ $271,281-284,287,289,295-$ $296,298,301,320,362,378$, $383,385-391,393-398,400$, $402,404,406-408,410,412$,
414-420, 422, 424-426, 428, $430,434,436,438,440,442$, 444-454, 456-458, 460-462, 464, 466-472, 474-478, 484$485,507,510-511,515,517-$ $518,520,523-524$

identity construction 472

identity politics $270,467,469$ 470

identity, American 81

identity, Bosnian Muslim in everyday practice 450

identity, collective 396

identity, cultural 415,485

identity, denial of 281,283 , 287,298

identity, ethnic $263,320,395$, $448-450,452,458,464$

identity, Muslim national in Bosnia 449

identity, social 64,395

ideologies, martial 515

ideology $28,32-33,47,49-50$, 61-62, 72-73, 80, 90, 94, 98, $101,120,122-123,138,164$, $185,221-222,224,226,320$, $390,393-394,398,403,415$, $420,425,451,457-458,467$, $471,477,488,510,515-516$, 518-520, 524-526

ideology of the firstcomer's primacy 222

Idomeneus 521

Ilaga Dani 114, 118, 136

Iliad 14, 214, 281, 285, 303, $387,397,400,424,431,502$, 515-521, 523-524, 527-528

Illerup, Jutland 229, 234

Ilongot 119, 132, 138

imaging the captured 284

immigration 123, 127, 176, 230

imperialism 30, 47

imprisonment 282, 288, 298, 301

Incas 223

indebtedness 397, 399

Inderøy 280, 319, 322, 327, 329

indigenous people/populations $65,67,109,119,128,130$ $131,197,402,470$

individual actor 67

individual graves 305

individual responsibility 437 , 441

individual, rights and obligations of $24,40,48,265$

individualism 36,396 individualist $33-34,85$

Indo-European 43, 64, 163

Indonesia 32,36

Indus 43

inequality $33,97,99,111,171$, $184-185,262,266,403,472$

inference 493,501

Ingelmark, B. 306

inhumation $152,156,279,306$, $322,346,489,492-494,499$, 501-502, 511, 516

institutionalisation, signs of 395

institutions, durability or durée of 395,397

institutions, egalitarian 16-17, $108,167,170-171,180,182$ 183

institutions, egalitarian, impact on war and exchange 167 $168,171,180,182$

Insubres 230

insult $82,173,179,196-197$, 244, 284-285, 287, 293-294, 298-299, 301, 360

interactionist view 213,263

international law 78, 86

international relations, theories of 113,133

intruders 279,487

Inuit 115,129

Iran $42-43,280$

Iraq $43,48-49,51,431,454$

Iregren, E. 414

Ireland $6,107,134,141,144$ $146,148-150,153-164,198$, $202,232,339,478,504,507-$ 509,512

Iris 523

Iron Age $43-44,65,71,147$, $158,161,164,212,229,234-$ $235,275-276,282-283,285-$ 293, 295, 298-304, 306-307, $318,322,328,336-338,340$, $395,486,491,493,500-501$, $503-504,507,516,527$

Iron Age, early $275,328,395$, 493,516

Iron Age, late $283,287,291$ 293, 298, 300-302

Iron Age, middle 288

Iroquois $106,123-124,126$, 218-219, 226, 419

irrigation 219-220, 226, 238, 240,254

Isandra 224 
Islam 49, 449, 451-454, 464, 466,468

Islamic fundamentalism 441

Islandmagee, County Antrim 149

Isle of Lewis, Scotland 153

Isle of Man 146, 148, 160

isotopic analysis 150

Israel 44-46, 51

Italy $72,111,154,163,230$, 233-234, 236, 420-421, 506, 508,511

Ithaca $135,139,257,270,521$

Ixil mythology 474

Jackson, S. 246,257

Jahweh 44-46, 48, 54

Jakab, J. 368,381

Janssen, J.J. 52

Japan 47, 49, 93, 401, 427

Jebel Qeil 285

Jelšovce $342,344,349,353$, $356,360,364-365,367,370-$ 372

Jenkins, R. 395,397

Jensen, J. 63

Jericho $42,142,159$

Jerusalem 233, 455

Jesus 41

Jewish 455

Johansen, Ø. 319

Johnson, G. 224

Julian, Roman commander 231

just cause $38-39$

justice $24,38-41,44-46,48,53$, $265,289,424,463$

Jutland 57, 60, 70-73, 229, 231, $234,311,420-421,490,492$, $501,504,513$

juvenal 290, 303

Jørgensen, A.N. 400

Kaahumanu 255

Kaba 255

Kabyle 526

Kachin 220-222, 224

Kahekili 250-251

Kahoolawe 248

Kalahari Desert 32-33

Kalaiopuu 251

Kalaniopuu 253

Kamakau, S.M. 251-252, 257

Kamano 84, 133

Kamehameha 248-251, 253, 255-256

Kamehameha II 255

Kant, I. 39-40, 54
Karadjordjevo 463

Karimojong 266-267

Karl II, the Bald 232

Karma 220

Kauai 248-249, 257

Kaulong 204

Kaumualii 249

Kavieng, New Ireland 203

Kayhausen, Schleswig-Holstein 289

Keawa-nui-a-'Umi 252

Keegan, J. 89-90, 93, 114, 426

Keeley, L. 26-27, 64, 66, 70, 76$77,85,106,149,511$

Keesing, R. 119

Ke-li'i-o-kaloa 252

Kelly, R.C. 171-173

Kemp, B.J. 52

Kent $185,232,292$

Kenya 266, 269

Kepele cult/feasts 169-170, 176, 178

Kettlasbrunn 279, 372-373, $376-377,380,382$

Kieta 203

Kilmahamogue, Ireland 154

kinship 30, 35-36, 73, 92-93, $120,123-126,130-132,139$, $164,228,238,241-243,248$, $255,257,263-264,269,320$, $326,355,398,485,502,518$, 528

kinship, segmentary lineage system 126, 168, 171

Kirch, P. 249

Kiriwina 78

Kissinger, H. 48-49

Kiwalao 251

Kleinman, A. 465

Klings, Germany 338

Knauft, B. 26, 77, 93, 398-399

knife see dagger

Knight, D. 331, 339

Knossos 516, 521

knowledge, basis for social success 207

knowledge, content of 477

knowledge, location of 469470,477

Knowles, C. 204

Knowth, County Meath 149

Knudsen, A. 447

Koch, K.-F. 119,121

Kolind, T. 70, 527

Kopytoff, I. 222

Koran 453-454

Korsør Nor, Denmark 143
Koš’any Culture 348

Kosihy-Čaka 343, 363

Kosovo 427, 429, 445, 510

Kost'any Culture 358

Kottak, C. 221

Krajina $388,391,429,433-435$, 437

Kristiansen, K. 60-61, 66, 156, 319-322, 326-327, 495-498, 500-501, 507-508

Kroměříž 342

Kuklick, H. 76

!Kung bushmen 33

Kuschel, R. 92

Kushites 284

Kuwait 48, 428

Kville, Bohuslän 62, 293, 488

Kwaio 119, 136

Kyaka 79,86

La Tène period $228,230,234$ 235

La Tène, early 234

La Tène, late 228,230

La Tène, middle 230

Lacey, R. 168, 177

Lagaip Valley, Enga 177

Lai Valley, Enga 178

Lakau, A. 173, 183

Lake Victoria 221-222

Lakemba 243

Lambot, B. 289

Lanai 248

lance $338,361,364$

lance, copper 364

land clearing 413

land, pressure on 150, 212

Landes, D. 265

landscape of power 516

Lang, H. 120

Langness, L. $83,84,86$

Langobardi 228, 231

Langston, J. 335

Lanhill 146, 162

Lapan 189-190, 193-197

Lapan, stories about 193-194, 196

Last, J. 157

Latian captives 285

Lau 189, 258

Laugerud, President 473

Laurion, Attica 290

Lausitz Culture 492, 494

law and order 135, 184-185, 204, 402

Lawagetas 401, 521-522

Le Morte d'Arthur 424 
Le Petit Chauvort, Verdun-surle-Doubs, Saône-et-Loire 292 leaders make war 214, 238

leaders, successful 233 leadership 26, 86, 97-98, 108$109,114,137,168,180,182$, 189, 192-193, 196, 222-224, 230-231, 233, 237-241, 248, $256,261,317,397,399-400$, $402,404,477,517,521$

leadership and prowess in battle 196

leadership, ideology of sacred 224

leadership, stories about 193194, 196

Leki Male, Poland 507

Lengyel 409

lesions 275, 305-307, 312-318, $322,325,327,406$

lesions, bone $305,307,313-$ $317,322,325,406$

lesions, skull 314, 316-317

Leubingen 507

Levant $41-42,55,142,162$

Leviathan $31,36,66,71,77$, $86-87,101,111,128,135-$ $136,138,339,420$

Lévi-Strauss, C. $25.30,120$

Lewis, A.B. 205-206

liberation struggle 47

Liberia 261-262

Liddell, D.M. 147

Liffs Low, England 151

limes 215, 232

Lindow Moss, Cheshire 288

linear B text/tablets 283, 401, 516, 521-522

linear ditch segments 336

Linear Pottery culture 404

Linkardstown, County Carlow 145,150

Linzgau 231

Lion temple, Musawwarat es Sufra 285

Lion temple, Naqa 284

lion, synonym of war hero 527

Lipan, Manus 193-194, 198

Little Poland 341, 349, 370

Littleton Drew 146

Livy 38, 300, 303

Lizot, J. 32, 116, 118

Llyn Cerrig Bach, Anglesey 283, 291-292

Locke, J. 263

Lockerby, W. 257

Lockwood, W. 451
Logue, P. 149

Long Island, PNG 175

Long Wittenham, Thames Valley 155

Longue durée 397,424

Lord's Resistance Army 266

Lorengau, Manus 203

Lorenz, K. 31

Los Angeles 36, 185, 270, 421, $427-428,431,490$

loss, sense of 453

Lou, Manus 190, 193, 196

Louis I, the Pious 232

Lowie, R. 402

loyalties, conflicting 130, 168

loyalty, oath-bound 401

Lugii 231

Luluai 203-204

Lusehøj 502, 504

Lusitani, Iberia 284

Lüneburg 494

Maasai 265

Macedonia 510

Macehead 387, 394, 406-407, $410,413,415,417,484$

Maček, I. 451, 465

MacGaffey, W. 262

Machiavelli, N. 263

Machiavellian 33, 172, 179

Madagascar 51, 224, 226

Madang 203

Mad'arovce cemetery 353

Mad'arovce Culture 358

Mae Enga $69,114,118-120$, $122-124,126,128,132-133$, 137,184

Maiden Castle $148,151,164$ 165

Main River 230

Majawil Q'ij 475-477

Maji area 267-268

Malaita 119

Malay 192

Malaysia 32

male bonding 398,410

male clubs, purposes of 398

male clubs/brotherhoods 387 , 397-398, 403

male cult 68,183

male domain 522,524

male dominance $410-411,414$, 417,420

male identification, crisis in 427

male point of view 68 male, boys $83,91,169,172$, $193,221,244,269,320,348$, $355,387,399,405-406,414$, $424,428-429$

male, elderly $144,146,406$ male, high-status, armed 414 male, low-status, unarmed 414 male/men, young 108,123 $124,142,144-145,147,151-$ $153,156,158,172,180,184$, $192,202,285,287-289,296$, 300-301, 310, 316-317, 331, $335,366,368,388,399,408$, $414,423-426,428-430,485$, 499,506

malehood, adult 405

maleness $62,208,404,415$

maleness, warlike 62,415

Malinowski, B. 27, 33, 75-84, 86

Malkki, L. 447

Malkovich, J. 438

Mallaha 41

Malmer, M. 63

Malmö 72, 428

Malnutrition 312, 325, 327, 377

Malo, D. 252

Malory, Sir Thomas 424

Malthus, T. 53

Mam Tor, England 157

Manacles 290-291

Manambu 122, 132

Mangan, J. 423

Mangyan 129-131

manhood, crisis in 427

Mani, Peloponnese 523

Manley, J. 158

Mann, M. 28, 52, 90, 93-99, 108,239

Mansell Collection 293

Mantaro Valley, Peru 220

Mantel, E. 289

Manuai, Manus 193-195, 198

Manus Culture 190

Manus Province 110, 187, 198

Maori 73, 151, 401, 424

Marcomanni 227, 230-231

Marcomannic Wars 228, 231

Marcus Aurelius 231

Marine Corps (US) 423, 430

Maring 118-119, 125, 127, 136137

Marne River 230

Maroboduus 228, 231

Mars 298, 523

Marshfield 337

Marsi 231 
Marsk Stig Andersen Hvide 317 martial strain 398,515

Marx, K. 31, 66, 221, 396, 477

Maschio, T. 207

masculine/masculinity 66,82 , $108,388,404,410,423,425$ $430,517,522-523$

masculinities, violent 428,517 masculinity, ideal 426,428

mass deportation 302

mass grave, Sandbjerg 6, 305, 316

mass grave $15,18,58,143$, $211,215-216,277-279,305-$ $310,312,316-318,322,337$ $338,368-369,372-373,487$, 502

massacre $6,143,161,300,304$ $307,309,311,313-315,317-$ 318

Massilia 297

material culture $7,11,14-15$, $18,61,69,72-73,204-206$, $208,232,282-283,285,290-$ 291, 295, 298, 301, 340-341, $343,385-386,394-395,399$, $412,415,417,419,481$, 483$488,490,492,494,496,498$, $500,502,504,506,508,510$, $512,515-516,518-520,522-$ 526,528

material culture as agent 483 material culture as source of data 483

material culture, ambiguity of 485

material culture, creates social relationships 484

material culture, creator of distinctions 484

material culture, durable 484 material culture, mediator 484 material culture, multifunctionality 485,487

material culture, silent form of discourse 484

material destruction $\quad 433-434$

material discourse $386-387,403$

material similarity 412

material transition 57

material, exotic 404, 409

materialist approach/interpretation 141-142

materialist attitude 526

materialist-functionalist approach 67

materiality $484,489,515$ matrilineal 124, 356, 398

Matupi 202

Maui 248-251, 253

Mauss, M. 25, 29-31, 33-35, 78, $81-83,119,191,196,448$

Maya code 470,478

Maya culture related to invented tradition 390, 477

Maya Indians 390

Maya past, ownership of 471

Mbuke, Manus 190, 194

McKenna, T.M. 443

McNeill, T. 90

McVeigh, Timothy 428

Mead, M. 32, 77, 81-82, 85, 190-191, 196

meaning $28-29,35,40-41,45$, $69-70,76,82,87,91-92,95$, $100,164,194,208,213,229$, $265,269,292,298,301,319$ $321,328,361,381,385,394$, $413,439,446,453,473,475$ $477,479,484,486,507,517$, $520,526-527$

Mecklenburg 234, 494, 498, 503-504

Medes 43

media manipulation $388,435-$ $436,442,444$

media messages, incorporated into personal narratives 443

media, authority of 442

mediation rejected 268

Mediterranean 41, 53, 105-106, $228,230,232-233,235,421$, $455,467,479$

Mediterranean, eastern 105-106

Meggitt, M. 118-120, 122, 168, $173,175-176,179,183$

Melanesia 33, 71, 87-88, 93, $101,106,108,128,136,138$, 184-185, 187, 191, 198-199, 205, 258, 386, 391, 448, 467

Meldon Bridge, Scotland 151

Melian dialogue 37

Melpa 79

men, aggressive 116,133

Mengen 202

mercenaries 228-236, 399, 425, 429

Mercer, R.J. 147-149

Mercia 337

Meroe 284, 304

Merovingian Empire 228-229

Merovingian period 236
Mesolithic 63, 73, 116, 132, $141,143,146,159,161-164$, $306,318,421$

Mesolithic, late 63

Mesopotamia 42-43, 55, 280

Messenia 401, 522

Messina 233

metallurgy $344,410-411,494$

metaphorical statement 394

metonymic statement 394, $407,485-486$

Mexico 102, 184, 259, 403, $427,471,478-479$

Michaels, W. 86

Middle Ages $38,236,307-308$, $310,312,317,322,430,483$, 517

Middle East $10,18,472,479$

Middle Farm, Dorset 337, 339

Middle Farm, Wessex 157

middle-range societies 26

Midlands 159,288

Mierzanowice Culture 355

Migration period, protohistoric 215

migration, forced 219,390 , 472,476

migrations $59,71-72,168,175-$ $178,183,205,215,228,258$, 319

migrations as military campaigns 215,228

Mikloucho-Maclay, N. 191-192 military $13,15-17,24,28,40$ $41,43,46-50,52,72,76-77$, $85,89-90,92-102,108,110$, $114,116-117,120,122-126$, $128,131-132,135,141,157$, $182,188,197,201,212,214-$ $215,218,220,223,225,228$ 229, 231-234, 236-242, 249 . $250,253-259,266,281,291$, 293, 296, 303, 317, 320-321, $326-327,387-388,391,398-$ $400,402,423,425-427,429-$ $431,462,472,503,515-516$, $518,521-522$

military apparatus, limited 225 military coalitions 233

military institutions, ideological basis for 387

military leaders 98, 240, 242

military organisation 13,28 , 89-90, 92, 94-101, 108, 188, $214,218,237-242,254-257$, $320,398-400,516$ 
military service $40,231,233$, 426

military societies $100,320,399$, 515

military strength $120,123-125$, 128,182

military superiority 17,123 , 126, 131

military technology 188,197 , 427

military, feminisation of 430

militia 389

militärische Demokratie 402

Milošević, S. 434, 463

Minoan 494

mission stations 202, 293

Mitanni 43

Mobutu 262, 266

Moche art 295

Moche material, Peruvian 283

Moche, Peru 283, 287, 295, 300,302

mode of production, Asiatic 221

Moka 79, 83, 87

Molokai 248

Momostenango 472, 476

Monaghan, J. 472

Monarchy 39-40

Mongols 51

Montagu Harbour 202

Montejo, V. 470-471

Morava River 342

Moravia 7, 13, 341-344, 346, $360-361,364,368,371-373$, $378,382,421,511$

Moravian Gate 341

Moravská Nová 356

Morobe 203

Morris, I. 516

mortality $73,114,116,121$, $124,197,322,414,528$

Moseley, H.N. 192

Moses 39, 208, 516, 520, 527

Mosse, G.L. 425

moulds, clay 157,494

moulds, solid 494

Mount Pleasant, Wessex 151

movement of objects and ritual 206

movement, Communist 47

movement, liberation 47

movement, nationalist 469

movements, rebel/guerrilla 96 , 262, 265-266, 475

movements, violent youth 269

Mozambique 262, 271
Mrozek, D. 423

Mucking 157, 159-160

Mujahedini 441,443

Mundugumor 82

Mundurucu 116, 124

Mungiki 269, 271

Murray, A.T. 527

museum collections 204, 208

museum collections, source of evidence 204

Museum für Völkerkunde, Basel 205

muslim $13,233,236,389,443$, $446,449-454,456-459,461-$ 468

muslim, Bosnian 466

muslimness, definition of 463465

mutilation $262,285,364,366$, 372

Mycenae 43, 51, 283, 485, 494, 510, 516-519, 527

Mycenaean period 516-517

Münster, K. 202

Myrmidons 519

Myth of the War Experience $72,425-426,431$

Mýtna Nova Vés $342,344,348$ $349,351,353,355-362,364-$ $368,370-372$

Mälardal type 328

Männerbunde 396-398, 420421,515

Møller-Christensen, V. 317

Nages 300

Nagyrev Culture 341

Namatanai 203

Napoleon 32, 35, 45, 425

Napoleonic Wars 77

narratives $85,178,180,196$, $203,386-387,389,391,423$, $433,436-437,439-441,443-$ $444,446-447,465,467,488$

narratives, of nationalism 440

nation $40,46,48,76,102,218$, $228,242,257,263,290,388$, $425,437,439-441,445,450$, $452,462,466,468,471$

national disambiguation 440 , 442

nationalism $7,50,71,75,101$, 388-389, 419, 429, 433, 435, $437,439-445,449-450,452$, 464-468, 471-473, 478-479

nationalism, Croatian 435 nationalism, dominant discourse $388-389,437,442$ 443

nationalism, exclusive 388 , 434, 449

nationalisms, post-Yugoslav $435,437-439,441-443$

nation-state $40,90,93-94,96$, $101,228,237,258,446,452$, $464,471,478$

native American 81, 479

Natufian 55, 142

natural state $25,29-31,39,46$, 93, 520

nature of Man 84-85

Nazi Germany 75

Neanderthals 305

Near East 24, 40-44, 46, 53, 55, 138

negotiation $38,125,180,244$, $287,327,396$

Nemeti 230

neo-evolutionist approach 63 , 67

Neolithic $6,41-42,53,55,59$ $60,63-65,69-71,73,107$, $116,141,143-154,158,216$, $219,275,306,323,336,391$, $402,404,410,484-487,492-$ 494, 507

Neolithic, early $41,63,143$ $151,154,158,161,306,382$, 391,512

Neolithic, late $63,65,150-154$, $306,323,391,410,492,507$

Neolithic, middle $60,70,149$, $154,306,404$

Neretva 462-463

Nervii, Gallo-Belgic polity 282

Nestor (of Pylos) 401, 519, 521

Netekamani, king 284

Netherlands 58, 72, 218, 226, $280,296,338,340,417,490$, 504

Nettleship, M. 217

network $34,94,96,110,130$, $139,153,169,178,187-188$, 190-191, 199, 204, 228, 457, 472,502

Neupotz 232, 280

Nevermann, H. 192

New Britain 202-208

New Guinea 5-6, 12, 14, 16-17, $27,68-69,73,75-88,93,101$, $107-108,110,113-114,118-$ $120,124-125,128,135-139$, 167-169, 182-185, 187, 191, 
198-199, 201-204, 206, 208, $385-386,397,404,466$

New Guinea, Highlands 68-69, 79, 82-84, 107-108, 113-114, 118-120, 124-125, 128, 168, $175,82,208,386,397,404$

New Guinea, lowland and island $68-69,107,191,202-$ 203

New Ireland 202

New South Wales 202

New Testament 41

New War culture 428,430

new wars 223, 261-262, 270

Nielsen, P.O. 63

Nigeria $269-270$

Nile Valley $42-43,284$

Nile Valley, Upper 42

Nimrod 507

Nitra Basin 342-344, 349, 353, 371-372

Nitra Culture 7, 341-344, 346$349,353-356,358-364,366$, $368,370-372,381$

Nitra-Čermáň $342,349,352$, 363

Nižná Myšl’a, Slovakia 369, 373,378

Noco 246

Nomads 15, 60, 121, 129-130, $132,224,234$

Nordbladh, J. 60-61, 66, 488

Nordic area $498,508,510$

Nordland 322,328

Norfolk 161, 291, 302

Normandy 233-234, 236, 426

Normans 227, 232-234

norms and rules of behaviour 397

norms and values, warlike 117 118,122

Norse Christians 275

North America 77, 98, 162, $240,248,320,400$

North American tribes 399

North Ferriby, England 159

North Sea 229

Northampton, Midlands 288

Northover, P. 332, 339

Northwest Coast Indians 486

Northwest Coast, America 132133, 486

Norton Bavant 146

Norby, Schleswig 500

Norway 7, 154, 319-322, 326$329,338,467,493$

Notgrove 144 notions of the past 390,477

Nubia 42, 284, 303-304

nuclear/atomic bomb 24, 47

Nuer $92,101,115,117,126$, $132,135-136,263,267,270$

Numancia 301

Numidian cavalry 294

Nydam boat 298

Næstved 6, 279, 305, 307-309, 311-312, 316-318

Oahu 248-249, 251, 255, 257

Obii 231

objects, ownership of 206

objects, ritually powerful 206

obsidian 190, 192-193, 195-

$196,206,404,406-407$

occupation $18,47-49,51,150$, $161,163,215,227-228,230-$ $232,234,253,318,326,400$, 428,465

Odua People's Congress 269270

Odysseus 486, 519-522, 527

Odyssey $285,424,515-517$, $519,521-522,524,527-528$

offering in hoards 394

offering, ritual 281, 410

offering, sacrificial 296, 402

offering, votive 485

offering, weapons 306, 394, $402,486,512$

Ofnet, Bavaria 143

Ogof-yr-Ychen cave 143

Oikos 523

Oklahoma City 428

Olausson, D. 410

Old Testament $41,44,46$

Olšava valley 342

Olujic, M. 447, 462

Oppenheimer, F. 97

opposition, Europe/Balkans 389

oppositional behaviour 436

oracle 44

oral history $183-184,197,253$

Orange, southern France 298

Orchomenos 516

order, moral $31-32,35,77$

order, religious 31

order, social 29-31, 34, 61-62, $77,83,86,93-94,133,477$, $518,525-526$

Oregon 428

Orgetorix, Helvetian chieftain 289

Orkney 145,162

ornaments, bone 348 ornaments, copper 348

Orosius 298-299

Orsett enclosure, Essex 148

Ortner, S. 90

Osgood, R. 154-156, 488

Oslonki 409, 420

ossuaries 299

osteological $162,275,300,322$, $327,341,364,368-369,376$, 381

osteological analysis 322,376

Other, the $7,24,35,37,40,46-47$, $49,51,53,62-63,87,89-90,93$, $98-99,101,106,109,117,124-$ $125,130,153,168-169,172$, $181,188-190,195,197,204$, 208, 213-214, 216, 221, 225, $229,231-232,234,239-241$, $246,249-250,252-253,262-$ $264,284,293,308,313,320$, $322,346,362,370,376,385-$ $386,389-390,398,402,410$, $414,420,430-431,435-437$, $448,459,462,467,471,474$, $476,485-486,488,491,493$, 495, 497, 499-501, 503, 507, $517,519-520,526-527$

Otherworld 287, 291, 299

Ottenjahn, H. 494

Otterbein, K. 23, 26-27, 66, 76$77,85,120,129,398-399$

Ottoman Empire 429

Ottonian 227

Over Vindinge, Præstø 61, 338

Oxford University Parks Science Area 152

Pacific, the $92,202,205,208$, $211,214,248$

pacification $6,14,17,87,106$, $110,113,117,120,127-130$, 136, 1.38, 187, 201

Painsthorpe 145

Palace of Nestor 401

Palaeolithic 41, 54-55, 141

Palermo 233

Palestine $41-43$

palisade $145,147-149,151$, $165,192,195,229,243-245$, 492

Pallas 285, 523

Pallas Athena 523

Palstave 493, 501

Pan-Mayan Movement 390

Papua 202, 208

Papua New Guinea $6,12,14$, $17,68-69,73,80,86-87,93$, 
107-108, 110, 135, 167-169, $175,184-185,187,191,199$, 201, 208, 385 see also New

Guinea

paramilitary groups $428-429$, 434

Parijs, P. van 218

Parioi, Manus 193-196, 198

Paris (France) 232

Paris (prince of Troy) 523

Parker Pearson, M. 336

Parkinson, R. 191-192

participation in decision-making 172

partisan 433,445

past reconstructed 110,187 , 202, 277, 307, 364, 413, 483, 521

pastoral tribe/societies 57,121 , 129, 132, 265-266, 399

paternalism, North American 471

pathology, pathological method $143,275,277,280$

patrilineal $120,123-124,130$, 189-190, 398

patriotism $13,39,45,50,389$, $431,453-455,464$

Patroclus 281, 285, 519-521

pattern of culture, Apollonian 81-82

pattern of culture, paranoid 8182

Patton, General 423-424

Pavlides, C. 204-206

Pax Romana 110

payback 175, 177, 196

payback as modality of reciprocity 196

peace $6,12,30-31,36,39-40$, $45-46,48,51,54,64-65,67$, 69, 75-76, 78-80, 82, 84-85, $87,91,96-99,107,109-110$, $113,115,117,119,121-125$, 127-129, 131, 133-139, 150, $169,172-174,179-180,183$, 185, 195-196, 216, 240-241, 249, 253, 256-257, 264-265, $267,270,282,317,319,326$, $393,397,402,421,426,441$, $452,461-462,465,469-470$, 472 , 475-476, 487, 489, 491, $521-523,525$

peace, cheaper to buy 216 peace, limited 51

peaceful 14-15, 17, 25-27, 32, $36,40,54,58-60,62-67,69-$
$70,72,76,80-81,87,93$, 106-111, 114-119, 121-122, 127-131, 133-136, 151, 162, $174,176,184-185,192,215-$ 216, 221-222, 305, 319, 386, $390,414,425,428,431,470$, $478,487,491,501,511,523-$ 525

Pearson, K. 78

peasant culture 63

peasant, peaceful $14,58-59,63$ peat-bog 296, 298-299

Peisistratos 527

Pelopides, Dynasty of 518

Peloponnese 523

Penelope 522

Pentagon 475

Penywyrlod, Wales 144

Perez, G. 473

performance $53,117,181,184$, $265,268,292,296,424,518$, 520

Pergamum 298

peri-mortem treatment 379

Persia 43-44, 47, 276, 296-297

Persia, Achaemenid 276

Persian Gulf 47

Persian Wars 296

personal qualities 192,387 , $397,399,409,518,521,524$ personhood, corporate 283 personhood, individual 283 personhood, surrender of 292 Peru 220, 295-296, 301

Pešić, V. 429

Peterson, D. 32-33

Petit Chasseur, Sion 506-507

Pezinok-Cajla 346

Phaiakians 524

Philippines $132,443,446$

Phillip, of Macedonia 230

phrygian caps 294

Pick, D. 465

Piggott, S. 63

pigs $119,124-125,136-137$, $169,173-177,179-182,184-$ $185,195,198,208,243,252$, 344

pig's-tusk ornaments 206

Pililo 206

Pilling, England 153

Pilum, Roman 156

Pitt Rivers Museum 12, 203, 205

Plague $305-306,313,326$

Plains Indian, North American 300,399
Plato $40,424,461$

Plavo, Croatia 433-434, 441442,445

Plutarch 295-298, 304

Poland $341,346,349,362,370$, $409,419-420,492,498,503$, 507

pole arms $153,361,364$

polis 518,527

political elite $15,262,443,451$

political unit, large $43,50,255$

political unit, small 14,43 , 114,217

politicisation, processes of 450 , 469

politics $11,14,24,28,35,46-$ $47,51,55,59,61,66,73,80$ $81,86,88,90,93-94,102$, $105,111,119,127,134,136-$ $137,139,168,175,178,183$, 198, 201, 225, 242, 254, 258, 261, 263, 266-267, 269-270, 385, 389-391, 402, 421, 431, 437-441, 444-446, 450, 457, 461-462, 464, 466-467, 469$470,473,475-476,478-479$, $490,526,528$

politics by other means 24,46 , 201, 385

politics of identity $390,445-$ 446,478

politics, danger of 438

Polybius 38

Polynesia 249, 258

Popol Vhu, Maya Quiché book of creation 473

Population density 52, 84, 114, $118,130,176,212$

Population/demographic growth 52-53, 212, 222, 267

Porsmose 494

Porter, B. 89

Pospisil, L. 120

post-mortem treatment 380

Postumus, Roman general 232, 300

Post-Yugoslav wars 435,442

potlatch 61,486

Potterne, Wessex 158

pottery $147,149-151,153-154$, 157, 159-160, 192, 230, 291, $307,373,378,404,410,414-$ $415,420-421$

Poulnabrone, County Clare 144-145

power 5-6, 13-17, 24-25, 31, 33, $38-48,50-51,53-55,57,59$ - 
$62,64,70-73,79-81,84,89-$ 91, 93-102, 105, 108-109, $111,113,115-117,119-123$, 125-131, 133, 135, 137-139, $150,164,168,174-176,178$ $180,189,192-193,197-198$, $201,204,207-208,212,214-$ $215,220,223-224,226,230$, 237-243, 245-249, 251-258, 262-265, 267-269, 271, 283284, 290, 293, 296, 320-321, $326-328,356,368,371-372$, 385, 387-389, 391, 393-394, 396-398, 400, 402-403, 406, $408,415,419-420,426,430$, $433,435,439,442,444,448$. $449,469-470,473-478,483$, 485-486, 493, 501, 515-516, 518-520, 522-526

power enactment 403,526

power field 402

power games 396

power, adjudicative 120

power, authority converted into 239

power, economic $90,95,100$, 254-255, 265

power, force $98,102,128,223$, 258

power, ideological 95, 100, 108, 255

power, imbalance of 79, 116

power, institutionalisation of 238

power, military $90,95-96,98$, 108,242

power, networks of $94-96,98$ 101

power, persuasion $16,110,525$

power, political $38,41,71,90$, 100-101, 108, 254-255, 258, 435,448

power, sources of $16,90,100$, $108,150,255-256,284,400$, $402,477,520$

powerlessness $7,130-131,389$, $433,436-437,439,444$

practice, level of 100

Prehistoric society, tales of 27 , 59,66

preservation, post-burial 278

preservation, post-excavation 278

prestige $13-15,55,76,108$, $117,122,128,142,153-154$, 157-158, 190-191, 197, 201, 207, 217, 220-222, 319-320,
$338,341-342,344,355,364$, $370-372,398-399,404,410$, $453,485,507,510,515,518$, 521

prestige goods $13,128,153$, $157,222,338,341-342,344$, $355,364,370-371,404,410$ prestige goods, control of 222 , $342,355,371$

prestige goods, copper 344 prestige goods, trade of 341 , 344, 370

Priestess, Cimbrian 297

Př́kazy, Moravia 365-366, 368 primates $32,35-36,134,138$

primordiality $25,29-31,33-34$, 263

Pringle, R. 119

prisoners' dilemma 122-123, 126-128, 131, 133, 196

prisoners of war/prisoners-ofwar/POWs 219, 275, 281287, 290-298, 300, 518

prisoners, Dacian 294, 300 prisoners, Gaulish 283, 298 prisoners, naked 291, 294-295 prisoners, noble 281, 295-296 prisoners, Nubian 284, 292, 294

prisoners, sacrificial 290 prisoners, Taliban 281-282, 302 process of distinction 396 process, level of 28

production monopoly, defended w. force 190

profession $69,395-396,484$ 485

projectile injuries 143,338

projectile point 144

property, private $39,45,238$

Przeczyce, Upper Silesia 511

Przeworsk-culture 230

psychological bond 93

Punic War, first 284

punishment $45,117,128,172$, 201, 286, 291, 298, 306, 308, 328,474

punitive raid 195

Pupu, Nitze 168

Pyecombe barrow, England 151 Pylos 401, 516, 521-522

Qaraniqio 255

Q'esla Kub'al 474

Queensland 184, 202, 208

Quidney Farm, Saham Toney, Norfolk 291, 302
Quiver 409, 418, 494, 520

Raaflaub, K. 527

Rabaul 202-204

Radcliffe-Brown, A.R. 70

radiocarbon analysis 332

radiocarbon dating 154,323

raid $72,82,92,111,162,192$, $195,217,234,278-279,327$, 397

raid party, assembling 92, 190

raiders, mobile 158

raids, punitive 95

Rambo 427

Rameses II 284

Randers 501

Randsborg, K. 486, 500

Rank 15, 18, 60, 64, 69, 189, 198, 222-223, 234, 245-246, $251,253,257-258,281,287$, $296,298,348,355,358,368$, $378,387,394-395,400-403$, $413-415,417-419,485-486$, 501,521

Rapa 219,222

Rapiers 153, 155, 160, 483, 505,510

Rappaport, R. 118

Rauto 202

Read, K. 82-83, 86

Read's Cavern, Churchill, Somerset 291

rebellion $6,44,92,223,231$, 252-253, 296, 305, 307-309, $311,313,315,317$

reciprocity, negative $196-197$

reciprocity, positive 196

reciprocity/reciprocal 15,29 , $32-35,37-38,45-46,48-52$, $54,81,98,122-123,131$, 133, 168, 191, 196-197, 214, $222,224,241,247,256-257$, 264-265, 269, 281, 327, 387, $397,401,403$

recruiting slogans 423

recruitment $118,127,133,202$ 203, 207-208, 396, 522

Red Sea coast 266

refugee 388,435

religion $12,40-41,45,50,65$, $199,246,252,254,262,264$, $298,319,387,424,445,449$ $454,459,464,466-468,470$, $479,485,526$

Renamo, Mozambique 262

repatriating research 390,478 
researcher, position of the 390 $391,469-472,477$

Resic, S. 66

resignation $389,437-438,442$, 444

resistance $92,94,110,119$, 130-131, 171, 208, 213, 233, $245,262,264,266,271,388$, $391,402,429,436,438,446$, $449,465,467,475-477,525$

resistance studies 262

resources, access to 142,168 , 212

responsibility $10,14-15,38,40$, $50,282,389,436-438,440-$ $441,444,472,501$

responsibility, abdication of 438

retaliate, necessity to 14,196 , 281

retinue $64,228,253,400,515$, 521

revenge $24,38-39,43,49,68$, $92,115,117-118,132,134$, $172-175,178,180,182,184$, 195-196, 217, 243, 248, 250, $268,276,281,285,308,317$, $320,327,434,466,477,494$, 523

Rewa 243, 246-248, 255, 257

Rhine 230-232, 235

Ribemont-sur-Ancre (Ribemont) $276,289,299-300$

Richards, P. 96

Riches, D. 91

right to self-defence 39

rights, over labour and products 171

rights, to be protected and defended by group 172

ringforts/ringworks 157-158, 162

risk $24,70,116,123,142,158$, $172,252-253,284-285,292-$ $293,436,447,497$

rites of passage/rite de passage $77,279,296,320,387,423$, 429,486

rites to unite the clan 173

Ritter Volcano 203

ritual authority 149

ritual behaviour $287,289,300$ 301,373

ritual burial $\quad 380-381$

ritual depositions of weapons 486

ritual killings $372-373,381$ ritual sphere 408,511

ritual war, ritualisation of 15 , $61,65,67,182,429$

ritual, alcohol consumption $397,410-411$

ritual, eating and drinking 397

ritualisation $6,182,281-282$

rituals and violence 325

rituals, post-battle 298

rituals, secret magic 68

rivalry $61,132,262,264,266$, $372,415,419,485,515-516$, $520,524-525$

River Soar, Leicestershire 156

Rivers, W.H.R. 78, 86

Robarchek, C. 130

Robb, J. 154

Robertson and Hernsheim 202

Robinson, M. 335

rock art $72,154,321-322,326$, 488-490, 505, 507-508

rock carving $285,287,295$, 321-322, 488

rock carvings, narratives of ideal nature 488

rock carvings, Scandinavian $287,295,321-322,488$

Rodmarton $144,146,337$

Roland 413, 424

Rollo 232-233

Roman Dacia 302

Roman Empire 38, 44, 105, 212, 215, 228-229, 231-232, 234-235, 295, 402, 470

Roman wars 283

Romania 508

Romanticism 62, 425

Rome 110, 230-231, 235, 276, $284,286,290,293-296,303$

Roquepertuse 300

Rosaldo, M. 132

Rosaldo, R. 119

Rousseau, J.-J. 24, 39-40, 45-46, $50,52-54,66$

Rowlands, M. 60-61, 66

Rudstone 338

RUF, Sierra Leone 262

Rusava valley 342

Russia 328, 358

Rygh, K. 322

Saale River 232

sacrifice $13,50,52,72,152$, $171,223,251,270,283,285$, 287-290, 295-299, 301, 303$304,320,340,342,344,390$, $425,430,445,470,490,504$ sacrifice for the nation 425

sacrifice, human/people 13, 15, $50,223,287-289,291,295$ -

297

sacrifice, weapon 61,276

sacrificial deposits 485

sacrificial victims to Dionysus 296

Saharan Republic 261

Sahlins, M. 68, 80-81, 119, 121, 252

Saka Valley, Enga 168,178

Sakkudei, Indonesia 32

Šal'a I 349

Šal'a-Dusikáre 363

Šal'a-Veča 352

Saladin 51

Salamis 296-297

Salerno 233

Sale's Lot 144

Salisbury Plain, Wiltshire 159, 336

Salisbury, R. 84

Samburu 265, 420

Samoa 36,202

Samurai 93, 303

San Bushmen 488

Sandauce 296

Sandbjerg 6, 277-279, 305, 308$314,316-317$

Sandin, B. 119

Sandwich, Lord 248

Saracens 233

Sarajevo 72, 454-455, 464, 467468

Sarn-y-Bryn Caled timber circle 152

Sau Valley, Enga Province 181

Saudi Arabia 49, 430, 454

Sava River 510

savage, brutal, bellicose, warlike 66-67

savage, myth of peaceful $26-27$, $64,66,70$

savage, noble and peaceful 26$27,64,66,70,76,80-81,84$, 242

savagery $28,75-77,80-81,86$, 285,460

Saville, A. 144, 147-149

Savory, H.N. 158

Saxony 234, 506

Saxony-Anhalt 229

Saxo-Thuringia 417

scabbards 499 
Scandinavia $16,70-71,73,164$, 277-278, 306, 321, 328, 413, $417,487-489,492,494$

Scania 492,498

scapegoat 293, 297

scarce resource, competition over $17,67,116,118,131$ 132

Scarry, E. 447

Schafstädt, Merseburg-Querfurt 417

Schela Cladovei 143

Schele, L. 471-472, 478

Scherrer, C. 132

Schleswig-Holstein 232, 289, 296, 502-503

Schletz, Austria 143

Schmidt, B.E. 320

Schnee, H. 191-192

Schröder, I.W. 320

Schwartz, T. 87, 190-191

Schwarzenegger, Arnold 427

Schweder, I.M. 377

Scotland 71, 144-145, 151-153, 155-156, 159-161, 164, 299, 303,412

Scott, J. 268, 388, 436

Scott, Walter 425

Scottish Highland clans 523

Sculptor's Cave, Scotland 156

security dilemma 122-123, 126$127,131,136$

Seddin $511-512$

Seine 233

Selec 346

self-defence $38-39,48,50-51$, 220

self-protection 202, 437-438, 444

Semai 32, 129-131, 134-135

Semai Senoi, Malaysia 32

Semnones 231, 290

Senones 230

Sepik River 71, 86, 184, 385

Sepik societies 182

September Eleven 281

Sequani 230, 235

Serapio/Agenarich 231

Serbia 49, 429, 431, 434, 446, 466-467, 510

Service, E. 96, 98-99, 119

service, for nation 425

settlement 41-42, 54, 63, 71-73, $119,121,128-129,143,147-$ $149,151,159-160,162-164$, $174,178,195,197,202,204$, 207, 215, 221, 229-232, 234,
288, 303, 327, 335, 341-342, $370-371,373,378-379,382$, $399,404,420-421,487,492$, $500,502,509-510,528$

settlement organisation and economy 371

settlement pattern, changed 204

settlement structure, shift in 370

settlement, defensive/defended $41,146,148-149,151,157$, 206-207

settlements, dispersed 492

settlements, location of 342

Shaka Zulu 236

Shakespeare 423-424

Shamshi-Adad 43

Shangri-La 33

Shanks, M. 527

Sherratt, A. $410^{*}$

shield, leather 154

shield, Nipperweise type 155

shield, silver 229

shield, wooden 154, 339

shield, Yetholm type 155

shield-bosses 229

shields, decorated 206

Shifferd, P. 225

Shinnie, Peter 284

Shorkaror, Kushite king 284

Siassi Islands 204, 206

Sibinii 231

Sicily 233

Sidbury Hillfort, Wiltshire 336 siege $42,72,158,245,295$, $317,467,523$

Sierra Leone 96, 102, 261-262, $265,270-271$

Sigersdal 493, 503

Sigmund, P.E. 38

signification $33,396-397,403$, 484,486

silences, telling 389,438

Silesia, Upper 511

Sillitoe, P. 120, 179

Silures 290

simple human societies 26,136

Single Grave Culture 60, 71, 421

Sion $506-507,512$

Sipes, R. 129

Sivo, Croatia 434, 445

Skalnìk, P. 257

skeletal evidence $151,158,286$, $325,336,493$ skeleton $57,143,145,147,152$, $154,163,278,288,305,307-$ $311,313-314,316-318,323$, $329,332,335,338,346,360$, $364,366,368,370,373,377$, $380,405,487,493,500$

skeleton, sex determination of 310

skeletons, pathological change in $316,323,373,376-377$, 380

Skeletons, Sund 277-278

skirmish $147,179,213,250$, 339,364

skull $41,58,143,145-146,150-$ $151,153-154,156,158,205$, $289,301,305-310,312-317$, $332,334-335,360,364,366$, $368,370,379-381,406-407$

skull/cranial fracture 150,360 , 364-366, 370

skull fracture, crush 364

skull fracture, depression 364

skull fracture, hack and slash 364

skull-pit 306

slave 192, 224-225, 243-244, $246,283,285,292-293$

Slovakia $7,13,231,279,341$ 344, 346, 348-349, 361-362, $369,371,373,378,382,492$

Smeeton Westerby, England 151

Smørumovre 500

social change $5,13,15-17,28$, $57,59-60,63,87,89-90,93$, 96-101, 107-108, 187, 198, 229, 239, 241-242, 247, 256, $370,393,398,402-403,446$, 487

social contract 25,31

social Darwinism 32, 133, 425

social field $61,251,396$

social organisation $28,30,94$, 96, 98-100, 123, 133, 204, 214-215, 228, 234, 238-241, $254,326,341,346,348,353$, $361,399,403,419,492,515-$ $516,518,520,524-525$

social position $133,326,346$, $385,393,399,484,518,520$ 521,524

social power $14,55,59,90,94$ $96,98-102,108,111,150$, $257-258,265,320,394,402$, $420,485,524$

social power, sources of 90,402 
social practice $14-16,28,57$, $67,69,73,86,90-93,341$, $394,402,404,409,419,516-$ $517,523,525$

social structure $11,16,67,86$, $90,98-99,110,204,240-242$, 247-249, 251, 256-257, 295, $309,325,353,361,371,381$, $396,405,409,413,415,417$, $419,483,487,525-526$

socialisation $117,120,122$, $133,264,452$

sociality, peaceful 32,81 societies, acephalous 106,122 , $167,170,265$

societies, age-graded 266,399

societies, barbarian 215

societies, Celtic 227

societies, clan $59,229,234$

societies, complex 99, 215, 222, $239,255,399$

societies, Germanic 227

societies, hierarchical 182

societies, male $397-398$

societies, matrilocal 130,402

societies, non-state $18,25,30$, $39,93,107,111,211,239$

societies, peaceful $63,109,129$ 130,133

societies, pre-industrial 398 societies, pre-state $26-27,105$, 237

societies, primitive $66,77,106$, 403, 419

societies, secret 397

societies, state $10,90,105,114$, 131-132, 261, 398, 402, 518, 527

societies, tribal $16-17,30,105-$ $107,113-116,118-121,123-$ $124,126-127,129-133,167$, $216,230,233-234,236,261$, $397,399,419,523$

societies, tribal without war $113,129-130$

societies, warlike $117-118,124$, $127-129,131,158$

societies, without central power $6,113,115,117,119,121$, $123,125,127,129-131,133$, $135,137,139$

society as biology 31 society as culture 31 society, big man 413 society, disruptive 65 society, ideal 483 society, level of 100 society, stratified $217,220-222$, $224,257,371$

sociobiology $31,35,116,133-$ 134,184

Sodbury Camp 337

Sofaer-Derevenski, J. 405

soldier $15,294,388,396,398$, $423,425,430,452-453,461$

soldier, mechanical cyborg 388 , 430

soldiers, female/women 388, 430

soldiers, professional 218,279 , 315,317

solidarity $82-83,95,120,123$, $126,171,180,182,265,397$ $398,415,453,464-465$

Solomon Islands 204

Somalia 261, 265

Sone, Manus 193

Sonna Demesne man 154

Sorabji, C. 451

Sorel, G. 263

source critical analysis/view $276,489,500,517$

sources which indicate violence 491

sources, historical 192, 215, $278,326,328,398-399$

sources, written $227,230,236$, $279,307,317,379,486,523$

South Africa 305

South America 98, 110, 240, $401,415,421,487$

South Cadbury, Somerset 160 , 287, 339

South Dakota 300

South Hornchurch 157, 161

Southeast Asia 221, 226

Southwark, London 288

Sovereignty $13,40,45,97-98$, $133,167,216,238,240$

Soviet Union $47-48,467$

Spain $110,227,301,402$

Spania Dolina 346

Sparbu 322,327

Sparta 37

spear $5,25,29,31,33,35,155-$ $156,158,175,179,190,192$, 194-195, 250-251, 288, 294, $332,334-335,338-339,368$, $493,502,508,511$

spear points, obsidian 190

spear tips, bone 493

spearhead $61,156,159,307$, $332-333,338,340,368,430$, $492,494,496,500,506-507$ spearhead, Bagterp type 494

spearhead, broad-bladed 156

spearhead, bronze $61,159,334$

spearhead, hollow-cast 156

spearhead, invention of metal age 494

spearhead, Valsømagle type 338

spearheads, Baltic 494-495

spearheads, Irish 494

spearheads, Minoan 494

spearheads, Mycenaean 494

specialist products 410

spectacle $286,290,292-293$

Speiser, F. 205-206

Spencer, H. 238-240, 256

Spinoza, B. 263

Spittler, G. 119,121

SPLM, Sudan 262

Sponsel, L. 32

sport $174,429,487$

Springfield Lyons 157,160

Springfield, Oregon 428

spurs, Mušov type 231

Sri Lanka 220, 222, 224, 226

Staines 145,163

Stamm 187, 228

Stammesbildung 228, 236

state administration 402,521

state and divinity 40,53

state and justice $38-41,44$

state dominance 110,411

state expansion (colonial) 106, 109,119

state formation $6,11,13,16$, 51, 97-99, 101-102, 105, 109, $212,214-215,217,219-221$, $223,225-226,237-243,248$, $255,257,259,261-263,402$, 471,511

state formation, factors leading to 212

state intervention 109

state power $39-40,44,105,113$, $128,214,257,262$

state structures, imposed 261

state, aristocratic 401

state, colonial 119, 201, 264, 402

state, early 223,238

state, pacifying effect 109,128

state, power monopoly of 119 , 128

state, Samurai-organised 401

state, sovereign $25,40,45,90$, 451 
states and tribal societies, coexistence of 234

states make war 214,223

states, birth/emergence of 52, 94, 97, 99, 109, 127, 219, 222-223, 227, 237, 239

states, postcolonial $48,80,262$ 264, 269

states, unstable 221

status $14-18,26-27,35,63,95$, $98-99,101,108,116,120$, 123-124, 137, 141-143, 147$148,153,158,170-172,189$ 190, 193-194, 196-198, $220-$ $221,224,234,243,245-247$, 249, 251-253, 255-257, 264, $266,277,279,281-283,285$, 287-289, 293, 295-298, 301, 306, 320-321, 327-328, 348, $355,360,368,373,376,380$, $387,394-395,397,401,403$, $405,414,417,425,429,449$, $464,477,484-485,501,507$, $511,515,517-521,524-525$

status and wealth, differences in 220

status competition/rivalry 17 , 141, 143, 196-197, 515, 520, 524

status, motive for war 196 status, warriors $387,507,518$ status, ways to enhance 252 253

Staxton, Yorkshire 152

Stead, I. 338

Sten Sture 307

Stepleton Enclosure 147, 149

Steuer, H. 400-401

Štiavnické Vrchy Mountains 346

Stillorgan, Ireland 154

Stocking, G. 76, 84, 86

Stolac, Bosnia Herzegovina 13, 389-391, 447-449, 452, 454465

Stone Age $14,36,57,59,61$, 63-64, 70-71, 87, 101, 164, 421

Stonehenge $146,150,152,154$, 160-161, 506, 512

Stora Vikers, Gotland 494

story lines 388-389, 439-441, 443-444

Strabo 294, 297

strategic essentialising 436

strategic interaction 107,120 121, 126-127, 131 strategies of disambiguation 441

strategies, repositioning 444

strategy, discursive 17

strategy/strategies $17,25,31$, $38,48-49,68-69,91,107$, $110,116,121-122,125-131$, $133,168,179-180,192-193$, $202,220,238,250,252-253$, $362,364,368,371-372,389$, $427,429,436,439,441-442$, 444, 463-464, 483, 485, 511, $517,519-520,524-525$

Strathern, A. 27, 79-81, 83-84, 86

Strathern, M. 27, 77, 80-81, 93, 191

strenght, moral 425

strenght, physical 425

structural approach 67,448 , 452

structural change $5,17,60,89$ $90,96-97,99,101,127,221$, 226, 241-242, 256-257

structural condition 121

Structural Functionalism 113

structural Marxist 60

Structuration theory 484,526

structure $7,11,16,18,67,71$, 73, 85-86, 90, 95, 98-99, 106, 110-111, 114, 119-121, 133, $136,148,163,182,198,203-$ $205,214,221,224,226,240-$ 242, 247-249, 251, 256-257, 295, 306, 309, 319, 321, 323, $325,327,329,341-342,346$, $348,353,355,361,370-371$, $378,381,395-397,401,405$, $409,413,415,417,419,437$, $442,460,473,483-484,487$, 501, 516-519, 521, 524-527

structure-agency approach 67 68

Subalterns 436

subjugation $6,96-101,215$, 219-220, 237, 239-242, 256 257, 281, 283-285, 287, 291, 293-297, 299, 301, 303

Sudan 261-263, 266-267, 270, 304

Suebi $227-228,230,235,290$

Suebi, confederation of 290

Sulka 202

Sund 277-280, 319, 322-329, 338

Suri $266-268,270$

surprise attack 245, 251
Sussex 148-149, 158, 160-161, 421

Sutton Veny bell barrow warrior 154

Swat Pathan 125

Sweden 62, 73, 287, 293, 300, 306-307, 318, 321, 417, 421, $424,428-429,461,492,504$

sweet potato, introduction of $167-169,176-177$

Switzerland 332, 417, 509-510, 513

sword 14, 72, 93, 146, 154-155, $158,162,297,314,321,324$, $326-327,484-485,487,493-$ $497,499-502,505,508,510-$ 511,520

sword-scabbard relationship 499 sword production 14, 494-495 sword, Ballintober type 156 sword, emergence of 487 sword, symbol of power 327 328

swords, bronze-hilted 494, 496498, 508

swords, cut and thrust 495

Swords, Ewart Park 508-509

swords, flange-hilted 321-322, 326-327, 495-498, 500-501, 508

swords, fully hilted 495,497 , 500-501

swords, griffzungenschwerter 508

swords, grip-plate 501

swords, Hallstatt 508

swords, hexagonal metal-hilted 496, 499

swords, import from Central Europe 495

swords, metal-hilted 496,498 499,507

swords, miniature 495,499

swords, objects associated w. 500

swords, octagonal hilted 495 , 498

swords, organic-hilted 508-509 swords, plate-hilted 496,499 swords, sharpening 321,488 , 496-498, 508

swords, signs of wear 497 swords, solid-hilted 508 swords, standardisation 495 swords, tanged-hilted 496, 499 swords, treatment 493,495 swords, vollgriffschwerter 508 
Sydney 198, 205

symbolism of submission 301

symbols of destruction and change 474

Syracuse 230

Syria $42-43$

Sørup 500

Tacitus 64, 227, 235, 290-291, 296-298, 397, 399-400

Tairora $84,88,120,123,139$, 185

Talheim, Germany 143,145 , 216,278

Taliban 281-282, 302

Tallington, England 154

Talyaga, K. 168

Tamboran cult 182

Tarrant Launceston 144

Tasman, Abel 242

Taylor, T. 291

Tchambuli 82

technological development 49 , 64,93

technological superiority 49

technology $7,15,17,28,42$, $49,90-91,93,96,100,102$, $110,167,179,188,197,205$, 212-213, 258, 267-268, 388, $427,485,487,491$

technology of war, innovations in 487

technology, new 17

Tedlock, B. 472

Tedlock, D. 476

Tee, Ceremonial Exchange

Cycle 79, 169-170, 176, 178, 181-182

Telemachos 521

Tepe Gawra 279-280

Terminator $7,387,423,425$, $427,429-431$

Terray, E. 217

territorial gain 142

terror/terrorism 47-51, 86, 115, $266,268,281,433,435,473$

Teutoburg Forest 279

Thames Valley 144-145, 148, $152,155,157-158,165$

theatre $60,292,296$

Themistocles 296

theories of (tribal) war 115-120 theories of (tribal) war, biological $115-116$

theories of (tribal) war, cultural 115,117 theories of (tribal) war, ecologi$\mathrm{cal}$ and economic 115, 118

theories of (tribal) war, historical 115, 119

theories of (tribal) war, political 115,119

theories, decision 121,133

theories, neo-realist 133

theory of role of war in Bronze Age 491

theory of war, reproductive 142

things, bearers of history and memory 520

Thirty Years' War 77, 460

Thomsen, C.J. 491

Thomson, E. 470

Thordemann, B. 306

Thornhill, County Londonderry 149

Thorsted 500

Thrapston, England 157

three-age system, Thomsen's 491

Thucydides 24-25, 37-41, 44$46,48-51,53-55$

Thuringia 230, 232, 339, 506

Thurnam, J. 146

Thurnwald, R. 78

Thwing, England 157

Tilley, C. 60

Tilly, C. $90,94,257$

Tilshead Lodge 146

Timpe, D. 231

Tinbergen, N. 31

Tiryns 516

Tiszapolgar 405-406

Tiszapolgar-Basatanya 404-407, 415,419

Tiszawalk-Kenderföld 407

Titan 188, 195

Tito 449

Točik, A. $341-342,370$

Todd, J.A. 205-206

Todorova, M. 459

Toke, M. 173, 175

Tolai 202-203

Toldnes $319,321-323,326-328$

tolerance $13,389-390,448-449$, $457-459,463-465$

tolerance, inter-ethnic 448,458

Toma, N. 473-474

tomb, chambered 144-146, 151

Tonga 242

tools for violence made exclusively as weapons 493

tool-weapons 404

Topol'čany 342
Tor enclosures 147

Tormarton 7, 156-157, 162, 277 $279,331,333,335-340,492$

Towton, North Yorkshire 306 trade, role of long distance 215 tradition, invented 390, 477

traditionalists $475-477$

Trajan, Roman Emperor 294

Trajan's column 294-297, 300

Transco 334, 339

trauma, facial 153

trauma, high frequency of 366 , 370,381

trauma/traumata $13,57,63,70$, $107,117,122,142-143,146$, 150-151, 153-154, 158, 208, $275,277,279,301,319,321$ $325,327,331,338,360-362$, $364-368,370,372-377,381$, 387-388, 395, 404-407, 409, $414,426,435-436,442,444$, $451,453,473,487-488,492-$ 493,508

trauma/traumata, skeletal 13 , $57,63,70,107,360,364$, $366-368,370,395,487-488$

traumas, World War II 388

traumata, direct evidence of violence 492

traumata, war-related 387,404

Treherne, Paul 411

Trelleborg 306, 318

Trenčin 342

Tribal Zone theory 67

tribalisation 228, 231-232, 234

Tribeč Mountains 346

tribes $6,15,26,29,57,64,68$, 80-81, 105-107, 109-110, $119,134,142,168-170,176-$ $177,180-181,184,208,227-$ $231,233-235,245,282,295-$ $298,341,397-400,402,404$, $419,500-501,521,523$

tribes, Arabic Bedouin 523

tribes, birth of 227

tribes, constitution of 234

tribes, Gallic 110

tribes, Germanic 110, 228, 297, $400,402,500,521$

tribes, Rhenish 296

tribes, Slavic 229

Triboki 230

tribute $93,98-99,132,219$, 231, 240-241, 243, 245, 247, 252-256, 258, 371

trinket, amber 417

trinket, bone 417 
trinket, copper 409-410, 414, 417-418

trinket, shell 417

trireme 296

Trivers, R. 35

Trobriand Islanders 79

Trobriands 81

Trojan War 38, 44

trophy skulls 143

trophy-taking $285,298,300$

Trotter, M. 311, 322

Troy/Trojans 51, 281, 285, 296, 519-523, 527

Trudshøj 501

Trundle 148

Trøndelag 322, 328

Tudjman, Franjo 434, 442, 463

Tulloch of Assery 144-145

Tultul 203-204

Tumu, A. 69, 119, 132, 168, 175,183

Tumulus Culture 338

Tupi-Guarani 106, 419

Turek, J. 414

Turkana 265

Turkey 51, 440, 449

Turney-High, H. 114

Turnus, Prince of the Latins 285

Turton, D. 133

Tutsi 228, 447

Tuzin, D. 180,182

Tvrdošovce $349,359-360,363$ $364,370,372$

Twr Gwyn Mawr, Wales 152

Ty-Isaf 144

Tymowski, M. 220, 222

Uganda 266,270

Ukraine 341,358

Umboi 203

Umi $102,249,252,259$

Umi-a-Liloa 252

UN missions 429

UN peacekeepers 429

understanding, inflexible 414

UNESCO 269, 455

Únětice Culture 362, 365-367

UNHCR 433

unification $40,46,51,228,451$

Upper echelon 414

Uppsala $12,72,270,307,315$ $316,318,329,420-421,467$. 479

Ur 43,503

Unfield culture $394,499,502$

Urnfield migration 491
Urnfield period $346,489,498$, 510

USA $4,24,46-49,51,430,460$

Ustaše $433-434,444-445$

Utilitarian 33, 156

Vadomar 231

Váh Basin 341-344, 349, 371

Vah River 343, 372

Valdemar Atterdag, King of Denmark 306, 317

Valdemar Sejr, King of Denmark 317

Valhalla 423

Valsømagle $61,338,494$

Values of civilisation 441

van den Berghe, P. 473-474

van der Dennen, J. 32-33

Vancouver, Captain George $248,250,254$

Vandkilde, H. 70, 297

Vangiones 230

Vanua Levu 242

Varna I 404

Varus-Schlacht 231

Velasco, M.A. 471

Velatice 511

Velim, Bohemia 337

Velké Žernoseky 509

Vel'ký Grob 342-343, 349, 362363,381

Vencl, S. 57,66

Vendel period 236

vendetta 447

Venezuela 32,69

Verata 243

Vercingetorix 286, 295, 298

Versailles Treaty 81

Vestfold 328

Věteřov Culture 373

victims $7,12,52,67-70,75,91$ $92,150,153-154,156,158$, 198, 213, 218, 287, 289-290, 292, 294-298, 305-318, 322, $331,335-339,368,376,378$, $380,389,405,436,446,448$, $461-465,467,476,479,488$, 502

victory $24,37-38,43-44,46-49$, $51,81,113,124,172,179$, 201, 220, 233, 243-244, 251, $266,296-300,397,431,486$

Victory, battlefield $48-49$

Victuales 231

Vietnam $47,55,66,72,423$, $426-428,430-431$
Vietnam War $66,72,423,426$ 428,431

Viking Age 215,306

Vikings 232, 236

Vikletice $414,416,419$

violence $7,11,13-17,23-33$, 36-39, 41-44, 46-47, 49-54, 57-58, 60-73, 76-78, 80, 82$87,89-92,95-96,99-102$, 106-107, 110-111, 113-115, $117,121-123,127-132,134$, 136-139, 142-146, 153-155, $157-165,172-173,179,184$, 191, 197-198, 202, 208, 211, 213-214, 216-220, 222-225, 237-238, 254, 256-258, 261271, 275-279, 282, 299-300, $303,305,318-323,325,327$ $329,335,338-339,341,364$, $366,368-369,371-372,376$, 385-387, 389-391, 393, 396$399,402,405-407,417,419$ $421,427-429,433-434,436$, $439,442-449,451-453,455$, $457,459-461,463-467,469$ $472,477-479,487,489,491-$ $494,506,512,520,523$

violence and counter-violence 115,128

violence and identification 7 , $13,72,447-449,451,453$, $455,457,459,461,463,465$, 467

violence and ritual 329

violence as idea 29

violence by colonial officers 208

violence, archaeological evidence 321-322, 492

violence, collective 92

violence, context of 278

violence, gang 214

violence, institutionalised 268

violence, inter-communal 53

violence, legitimacy 91-92, 478

violence, meaningful/meaning of $28,67,69,91,321$

violence, monopoly on 208 , $214,237-238,261,267$

violence, non-political 469

violence, organised 52-54, 95, $100,217,257,320,366,402$, $469,491-492$

violence, physical $57,82,89$, $91,92,96,100-101,173$, 393,487 
violence, political 47, 51-52, $100,138,262,265-266,268$

violence, rites to manhood 427 violence, ritual activity/ritualised $115,213,262,320$ 321

violence, sacred 299

violence, structural theories of/approaches to 448,452 , 464

violence, threat of $16,50,402$ violence, visible to senses 91 violent behaviour, valued 117 118

violent displacement 433

Virgil 285, 304

Visby, Gotland 306, 310-311, 317

Vistad, Östergötland 492

Vithikap 231

Vitiaz Strait 203

Vladár, J. 361

Vlasac 143

Voldtofte, Funen 492

Vukovar 443, 445

Vỳapy-Opatovce 342,344 , $352-353,359,362-363,370$ 371

Vyšnỳ Šliac 509

Völkerwanderungen 228

Völkerwanderungszeit 231, 504

Waddell, E. 168

Wagner, R. 79, 119

Wainwright, G.J. 151

Wales 12, 143-144, 146, 151$152,157-158,161,202,283$, 290-292, 303-304

Wallace, S. 86

Wallis, M. 257

Wanax 401, 420, 519, 521

Waorani 116, 118, 124, 128, $133,136,138$

war against the state 106,419 war and absolute force 38,41 , 49

war and absolute justice 41,45 war and anthropology 86, 466 war and archaeology 73, 139, 490

war and areal integration 187, 196

war and central control 223

war and destruction 24, 43, 47$51,54,83,116,118,126$, $180,195,218,248,390,433-$ $434,456,460,463,492$ war and glory $46,385,396$

war and loss 443

war and music 205

war and non-state societies 18,

$93,107,111,211$

war and punishment 328

war and rationality, rationalisa-

tion of 54, 69-70, 81, 196

war and reproduction of society

$90,110,404$

war as a way of life 229

war as context $83,238-239$, 255

war as custom 80,308

war as erklären 67

war as exchange/transaction

17, 25-26, 108, 170, 180-181,

191, 196-197, 201-202, 207

war as field $7,469,471,473$,

$475,477,479$

war as means to increase politi-

cal power 239-240

war as part of state concept 211

war as policy 40,47

war as practice $5,89-93,95,97$, 99, 101

war as process $16,96-100,109$, $238,255,404,434,477$

war as rite de passage 387

war as ritual practice 320

war as site $7,469,471,473$,

$475,477,479$

war as social action 59, 69,

$106-108,393,404,486$

war as social duel 79

war as strategy/strategic 14, 16, $33,67,69,120-121,131$, 220,253

war as structure $67,85,180$, $182,248,254,256-257,321$, $327,371,419$

war as verstehen 67

war chief/leader $64,79,98-99$, $142,171,181-182,189,197$, $214,218,230,238,240,242$, $244,254,276,286,298,320$, $327,387,393,396-397,399$ $403,483,521$

war chiefs, female 142 war creates groups 14, 448

war criminal 429

war hero $321,517,519-520$, 523,527

war lords $6,216,227,229,231$ $233,235-236,265,285,400-$ 401 war made/make states 212 , $214,237,247,255,257$

war magic 4,386

war of the gift $77,80-81$

Wars of the Roses 306

war on terror 49

war propaganda 442

war raid/expeditions $62,75,90$, 124, 222, 229-231, 233-235, 243-244, 279, 320, 327, 361, $372,397,399,404-405,417$, $488,501,523$

war reparations $169,174-176$, 181-182

war rituals $18,117,122,132$, $320,341,385$

war symbolism/symbols/images of $79,151,285,294,300$, $409,426,488$

war tools 413

war veterans 426-427, 429

war veterans, Vietnam $426-427$

war, analysis of $28,37,75,77$,

$86,90,93,100$

war, anarchic 82,122

war, ancient $53,73,251$

war, armed conflict between groups 115, 229

war, artefacts designed for 319 war, beginnings of 42

war, Biblical 51, 54

war, causes of/reasons for 17 , $53,67,116,118,120-122$, $126,133,173,175,183,222$, $234,243,327,470$

war, ceremonial, to preserve benefits without destruction 180

war, civil $77,113,155,266$ $267,317,390$

war, civilised 113-114

war, collective side 320

war, conceptions $24,26,38$, 100

war, constant/permanent state of $117,119,121,126,128$, 267,402

war, conventional 92,108, 180, $182,250,487$

war, declaration of $39,91,253$

war, defensive 40,51

war, definition (of) 44, 90-91, $115,132,201,213,217$

war, driving force in evolution, (social) evolution $27,89,96$, 222,238 
war, egalitarian constraints on

$168,173,175,180,182$

war, elements of 213-214

war, endemic $44,77,106,128$, 218,523

war, ethnic/ethno-political 15 , $67-68,113,228,472$

war, explanation for centralisation 98, 214, 220, 239-240, 254

war, explanation for social complexity $99,106,239,241$ 242,255

war, explanation for subjugation 97-98, 239-240, 256

war, external $124,174,177$

war, forces clan societies to (be) tribes 234

war, generating complexity, hierarchy 167

war, given up 204

war, homeostatic function 106 , 167

war, iconographic presentations of 57

war, ideological $24,47-48$

war, increased 404,487

war, independence 113

war, indigenous $16,119,131$

war, individual side 320

war, just/moral $24,38-41,45$ $46,48-50,53,220$

war, justification of 24

war, legitimate 461

war, limited 48,51

war, logic of 16,450

war, low intensity 261,267

war, makes groups 386

war, spoils of 196-197, 229, $245,290,298-300,387,397$, 400,518

war, meaning of/interpretation of $54,69,78,85,321,385$

war, modern $27,76,82$

war, non-state $10,18,93$

war, nuclear 47

war, organisation $13,89-90,94$, $108,123,214,237,256,404$

war, organisation of in rise of states 211

war, origin $23,31,41,65,141$, 448

war, outgrowth of disputes between individuals 173

war, owners of the 180-181 war, part of basic social norms 208 war, part of reproduction of social structures 99,240

war, philosophy of $18,37,44$, 53,80

war, planned organised armed dispute 23,114

war, playful form of fight 114 war, postcolonial 113

war, precolonial $187,191,193$

war, primitive $66,106,111$, 419

war, prowess in $173,194,196$, 246, 399, 404

war, purpose/aim of $38,48,49$, $116,180,212,404$

war, restricted $47,114,127$, 182

war, rooted in biology 69

war, savage $27,75-79,82$

war, scale of 69,107

war, signs of 408

war, small-scale $43,90,279$

war, sociality 32,81

war, stories about 193

war, technology $90,179,388$, 487

war, total $27,47,76-77,80-85$, 425

war, tribal $28,71,76,111,114$, $119,121,126,129-130,135$, 184

war, ubiquity of 187

war, unrestricted 182

war, used to defend specialisa-

tions 197

war-captives 282-286, 298-299

Warder 292

war-games 108

Warku cult/ceremonies 206-207

Warku masks 206

Warne, C. 150

Warre 5, 25, 29-31, 33, 122, $215,218-219,500$

Warren, K. 469, 475, 478

warrior as masculine ideal 7 , 423

warrior band/war band 14,158 , 215-216, 227-234, 338, 387, 397-400, 403-404, 408-409, $415,515,521$

warrior bands, formation of 233

warrior bands, mobile 158 , 228, 230-231

warrior burials $347,355,368$, 402 warrior clubs $15,108,398-399$, 401-402, 415, 417

warrior equipment 153,348 , $354-355,500$

warrior equipment, in children's graves 355

warrior identities and material discourse 386

warrior identities and narratives (of) 387,391

warrior identity $15,215,378$, $394-396,400,419,484-485$, 510-511, 515

warrior identity, understood in social context 515

warrior institution and membership/access $15,320,387$, 397 , 399-400, 404, 415, 521522

warrior institutions $7,15,18$, $386,393,395-397,399-403$, 405, 407-409, 411, 413, 415, $417,419,421,521-522$

warrior institutions, age graded 402,409

warrior mentality $423-424$

warrior myths 391

warrior nostalgia 428,430

warrior society/societies 320 , $327,402,425,427$

warrior sodality 415

warrior tales 387

warrior values $17,72,387-388$, $391,394,424-429,431,485$

warrior, Aztec sun 401

warrior, boundary crosser 396

warrior, chiefly 402

warrior, common/ordinary 348 , $350,355-356,358$

warrior, cyborg 427,430

warrior, doubleness 396

warrior, elite/high-ranking 64, $348-349,355-358,360,362$, $368,370-372,417,428,485$, 488,511

warrior, female $64,142,355$ warrior, horse-mounted 62 warrior, idolised/idolising of 66-67

warrior, on ship 62

warrior, organisation 15,353 , $355,386-387,397,402$

warrior, person who used weaponry 506

warrior, professional 316, 399400 
warrior, specific male identity 515

warrior, tribal 122

warriorhood and life cycle 395$396,399-400$

warriorhood as daily routine activity 396

warriorhood as identification 395

warriorhood as negotiated difference 396

warriorhood as relational and interactive $395-396$

warriorhood, celebration of $388,391,426$

warriorhood, construction of 396

warriorhood, institutionalisation of $18,387,393,396-397$, $402,404-405,408,486$

warriorhood, material symbols of 351

warriorhood, non-institutionalised 397,404

warriorhood, revival of 425

warrior's gear, children and women with 355

warriors, aristocratic 60,397 398, 401, 518-521, 524-525, 527

warriors, buried in pairs 358

warriors, classes of 348

warriors, function in constitution of tribes and states 234 warriors, social organisation of 353

war's creative role 212

wars, causes and effects of 6667,227

Wars, Great Ceremonial 108, $169-170,178,180$

war-veteran clubs 429

war-victims, sacrificial 285

Wassenaar $58,72,213,216$, $218,226,278-280,338,340$, 490

Waterhouse, J. 257

Wayland's Smithy 144-145, 159,165

wealth $35,76,78,80,93,126$, $128,147,168,170-171,174-$ $175,179-182,190-191,195$, 206, 220, 236, 241-242, 247, $254,264,270,319-320,348$, $356,370-371,404-406,409$, $411,413-414,451,484,488$,
$492,500,502-503,518,520-$

521,524

weapon arsenal, source for war 493

weapon carrier 405

weapon combinations $485-486$

weapon finds 386,509

weapon technology 15,213 , 485,487

weapon, internet as 427

weaponry in graves/burials 321, 361-362, 394, 485

weaponry in votive deposits 485

weaponry, defensive 493,505

weaponry, destination of 485

weaponry, elite 507

weaponry, frequency of 64 , $342,370-372$

weaponry, masculine expression 522

weaponry, offensive 493,502 , 505-506

weaponry, prestigious 107

weaponry, social marker 361

weaponry, social role of 483

weaponry, used for chronology 361

weaponry, visibility of 402

weapons import 214

weapons in sacrificial deposits 485

weapons make warriors 484

weapons of mass destruction 48-50, 427

weapons, blunt $143,146,151$, $313,362,364-365,368$

weapons, close combat/close quarter fighting 114,253 , 319, 361-362

weapons, destroyed or damaged 155-156, 276, 299-300, 321, $496,508,510$

weapons, function of 505,508 weapons, have biographies and stories 486

weapons, iron 276

weapons, long-range 14,361 , 502

weapons, maintain identities 485

weapons, metal $319,321,344$

weapons, new 197, 254, 268

weapons, personal objects 410 , 486,510

weapons, sign of participation in combat 234 weapons, signifying rich grave 502

weapons, single, placed in wet areas 500

weapons, specialised 394

weapons, spoils of war 245

weapons, technological

character of 49

weapons, tools of war 484

weapon-tools 404-406, 408

Webb, M. 215, 239, 241-242

Weber, M. 66, 132, 263, 265

Webster, D. 241-242

Wedgwood, C. 78,86

Wees, H. van 515

Weiner, A. 33

Weland 232

Werner, J. 232

Wessex 144-146, 150-152, 154, 157-158, 160-162, 164, 336$337,339-340,494,512$

Wessex Culture 158, 161, 512

West Kennet, Wessex 144

West Tump 144

Westermarck, E. 78, 86

Wetterau 230

Whitehawk 148

Whitehead, N.L. 67,119

Whitley, J.' 485

Whittle, A. 149

Wiermann, R. 413-414

Wiessner, P. 69, 108, 119, 183

wife stealing $115-116,118$

Wilburton period 509

Wilkes, C. 257

Williams, T. 243, 247

Wilson, E. 31, 141

Wilster, C. 527

Windeby, Schleswig-Holstein 296

Windmill Hill 149, 165

Windy Dido, Hampshire 336

Winkler, E.-M. 377

Wissey Embayment, England 154

Withington, England 153

Witu 203

Wohlt, P. 168

Wold Newton, Yorkshire 144

Wolf, E. 472

Wollmesheim 511

women see female

Woodeaton, Oxfordshire 299

Wor Barrow 144

world Cultures Database 398

world system 105, 113, 119, 131 
world system, formation of 119

World War I/the Great War 76$77,81,86,425,427$

World War II 27, 302, 319, 388, $425,429,431$

Wormsley, W. 173, 175

Worsaae, J.J.A. 491

wound 61, 143-146, 150-151, $154,156-157,250,289,306$, $313,317,325,331-335,338$, $361,364-366,368,372,376$, $405,487-488$

wounds, battle/combat 156, $313,331,338,361,364$

Wrangham, R. 32-33, 142

Wright, Q. 77, 90

wristguards see bracers

wrist-shackles 291

Wüstemann, H. 506
Xingu 130-131, 135, 137

Xingu Basin, Upper 130

Yaghan 115,129

Yanomami 32, 35-36, 69, 71, $98,106,110,114-120,123-$ $126,133-137,142,218,223$, 240, 419-420

Yde, Netherlands 296

Yesner, D. 133

York, J. 155-156

Yorkshire 144-145, 150, 153$154,156-157,306,338$

Young, D.W. 183

Yugoslavia 17, 386, 389, 429, $433,438,445,447,449-451$, $455,461-462,508,510$

Yugoslavia, ex- 268, 386, 389, $438,445,447,461,509-510$

Yugoslavia, socialist 429,445
Zagreb 431, 445, 465

Zaire, ex- 265

Žanić, I. 429

Zealand $61,143,307,317,391$, $424,494,498$

Zeleno, Croatia 434-435, 445

Zenica 454

Zimmerman, L. 300

Žitava River 342-343

Zuiderwijk, A. 222

Zulu 228, 236, 268, 401-402

Zumi 231

Zuni 81

Æbelholt Monastery 314, 317

Östergötland 329, 492

Østfold 322

Ötzi 277, 506 